\title{
Towards accurate cosmological predictions for rapidly oscillating scalar fields as dark matter
}

\author{
L. Arturo Ureña-López ${ }^{a}$ and Alma X. Gonzalez-Morales ${ }^{a, b}$ \\ ${ }^{a}$ Departamento de Física, DCI, Campus León, Universidad de Guanajuato, 37150, León, \\ Guanajuato, México. \\ ${ }^{b}$ Consejo Nacional de Ciencia y Tecnología, Av. Insurgentes Sur 1582. Colonia Crédito \\ Constructor, Del. Benito Jurez C.P. 03940, México D.F. México \\ E-mail: lurena@ugto.mx, alma.gonzalez@fisica.ugto.mx
}

\begin{abstract}
As we are entering the era of precision cosmology, it is necessary to count on accurate cosmological predictions from any proposed model of dark matter. In this paper we present a novel approach to the cosmological evolution of scalar fields that eases their analytic and numerical analysis at the background and at the linear order of perturbations. The new method makes use of appropriate angular variables that simplify the writing of the equations of motion, and which also show that the usual field variables play a secondary role in the cosmological dynamics. We apply the method to a scalar field endowed with a quadratic potential and revisit its properties as dark matter. Some of the results known in the literature are recovered, and a better understanding of the physical properties of the model is provided. It is confirmed that there exists a Jeans wavenumber $k_{J}$, directly related to the suppression of linear perturbations at wavenumbers $k>k_{J}$, and which is verified to be $k_{J}=a \sqrt{m H}$. We also discuss some semi-analytical results that are well satisfied by the full numerical solutions obtained from an amended version of the CMB code CLASS. Finally we draw some of the implications that this new treatment of the equations of motion may have in the prediction of cosmological observables from scalar field dark matter models.
\end{abstract}

Keywords: Dark Matter, Cosmology 


\section{Contents}

1 Introduction $\quad 1$

2 SFDM background dynamics $\quad 3$

2.1 Mathematical background 4

2.2 Early, intermediate, and late time behaviors 5

$\begin{array}{lll}2.3 & \text { Initial conditions } & 7\end{array}$

2.4 Modifications in CLASS 8

2.5 Numerical results for the background 9

3 SFDM linear perturbations $\quad 10$

3.1 Early time behavior $\quad 12$

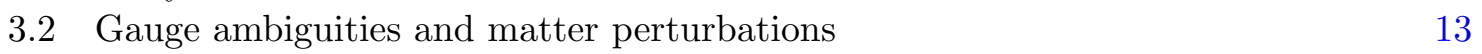

$\begin{array}{lll}3.3 & \text { Modifications in CLASS } & 14\end{array}$

4 Suppression of power in scalar field perturbations $\quad 16$

4.1 General form of the equations of motion for scalar field perturbations 17

4.2 General solution of perturbations after the onset of the field oscillations $\quad 17$

4.3 Large scales $k \ll k_{J} \quad 20$

4.4 Small scales $k \gg k_{J} \quad 22$

5 Power spectrum and mass function 23

6 Conclusions 28

$\begin{array}{ll}\text { A Cut-off procedure for rapidly oscillating terms } & 29\end{array}$

$\begin{array}{lll}\text { A.1 Background variables } & 29\end{array}$

A.2 Numerical examples 31

A.3 Linear perturbations 32

\section{Introduction}

The most recent observational results from the Cosmic Microwave Background (CMB) give strong support to the idea of a benchmark model of the Universe that is consistent with a variety of observational data[1]. It is remarkable that this standard model, well known as the $\Lambda \mathrm{CDM}$ model, is at its core just described by six numbers with a true cosmological context; the rest of the physical quantities and constants, which are important for a complete understanding of the high and low-energy processes in the evolution of the Universe, can be fixed and measured by other methods at terrestrial laboratories.

One of such cosmological numbers is particularly important to understand the formation of cosmological structure: the actual amount of cold dark matter $(\mathrm{CDM}), \Omega_{c} h^{2} \simeq 0.119$. Dark matter represents about $27 \%$ of the total matter budget. The base model for CDM is as simple as it gets: a pressureless fluid of particles that have no direct, weak at most, interaction with other matter fluids apart from the gravitational one. These two basic properties are enough to provide a complex picture of structure formation through hierarchical assembling that fits 
data at different scales[2,3] (see also[4] for a historical account of dark matter). A detailed model of CDM is necessary, but so far observations seem not to be very demanding in that regard[5-8], and a direct detection of the dark matter particle in terrestrial laboratories has so far produced only null results, though significant progress is being made [9-11].

A satisfactory answer to the CDM riddle have to be complemented with a convincing theoretical model that give us a comprehensive understanding of its properties. Although there is quite a large number of proposals, here we will focus in a type of model that has been studied for more than two decades: scalar field dark matter (SFDM)[12-29]. The key concept in this model is that the dark matter properties can be described by the dynamics of a scalar field with a probably fundamental origin, and a very tiny mass of around $10^{-22} \mathrm{eV}$, as suggested by different cosmological and astrophysical constraints. Cosmological scalar fields are better known in the context of dark energy models because many proposals are able to provide an accelerating expansion of the Universe at late times[30]; however, for dark matter models it has been widely shown that any successful model must be at the end related, somehow, to the simplest possible case: a scalar field potential with a parabolic shape around a minimum (see $[31,32]$ for comprehensive reviews).

The typical form of the scalar field potential in SFDM models is:

$$
V(\phi)=(1 / 2) m^{2} \phi^{2},
$$

where $\phi$ is a real scalar field, and $m$ is its mass scale. As we said before, other potentials exist for SFDM models, but their common feature is that they all can be approximated by the functional form (1.1) once they enter the dark matter phase, see for instance[15, 17, 19, 23, 29, 33] (although higher order corrections to the potential can play an important role in other high and low-energy processes[24, 31, 34, 35]). The resultant behavior of scalar fields as a pressureless fluid appears from their rapid oscillations around the minimum of the potential (1.1), which is a well-known fact[36-38]. For rapid oscillations we mean that the period of the field oscillations, represented by the time scale $m^{-1}$, is much shorter than the typical Hubble time $H^{-1}$ for the expansion of the Universe, where $H$ is the Hubble parameter. Because the Hubble parameter is a decreasing function of time, the ratio $\mathrm{m}^{-1} / \mathrm{H}^{-1}$ quickly becomes very small for realistic models, and then accurate numerical methods are required for a precise evolution of the equations of motion[32]. This difficulty has been overcome somehow by the use of approximate formulas, see for instance[21, 23, 29, 37, 39, 40], that are intended to match as smoothly as possible the transition towards the pressureless phase of the scalar field dynamics (see also[41]).

As for any dark matter model, the properties of SFDM models must also be connected to the formation of structure in the Universe, and then the same physical properties that allow a consistent evolution at cosmological scales must also be appropriate for the development of realistic galaxy halos. The latter have been also extensively studied in the literature for SFDM models, see for instance[42-46, 46-50] and references therein. More recently, complete wave-like simulations have shown the similarities of SFDM with CDM[20,51] at large scales, although most of these studies have been done under the non-relativistic approximation. One another quantity that plays an important role here is the mass Power Spectrum (PS), which represents the aggregation properties of matter at different scales, whose measurement are for now in good agreement with the theoretical expectations of the $\Lambda$ CDM model if the scalar field mass is large enough. Nonetheless, the mass PS is sensitive to the physical properties of the dark matter particles, and there may be noticeable consequences in the history of galaxy formation if the dark matter component is not strictly cold, as has been shown in[19, 52-56]. 
Given the interest in SFDM models in the recent literature, it is desirable to have an improved handling of their cosmological dynamics, for variables in the background and in linear perturbations, even if eventually one has to use numerical methods to resolve them completely. Along these lines, the aim in this paper is two fold. First, we would like to present a new approach for the solution of the cosmological equations of SFDM, so that we can have a proper understanding of the subtleties in their evolution. This will be achieved through the use of the tools of dynamical systems (for some pedagogical presentations see[57-59]), together with appropriate changes of variables that are more than suitable to exploit the intrinsic symmetries of the equations of motion. The techniques developed here can be easily extended to different cosmological settings in which scalar fields may be involved. Second, we will analyze the mass PS of SFDM models for different masses of the SFDM particle draw some implications on other observables such as the mass function, in a approximate way since the scales of interest are beyond the realm of linear perturbations.

A brief summary of the paper is as follows. In Sec. 2 we explain the conversion of the equations of motion for a scalar field dominated Universe into an autonomous dynamical system, where the latter is written in terms of a kinetic variable and two potential variables. Taking into account the symmetries of the system of equations, we propose a change to polar coordinates that helps to convert them into a more manageable 2-dimensional dynamical system that can be solved numerically without any by-hand manipulations. As we shall show, the new method is able to follow the evolution of the system from the early slow-roll regime to that of rapid oscillations with high precision and minimal numerical effort.

In Sec. 3, we apply similar mathematical manipulations to the equations of motion of linear perturbations, so that their evolution is also given in terms of a 2-dimensional dynamical system. All the new equations, for the background and for linear perturbations, will be put into an amended version of CLASS[60], which will be used to calculate all relevant cosmological quantities. We will clearly identify, within the new system of equations, the socalled Jeans scale of SFDM. As a side result, the velocity gradient is also calculated to verify the appropriateness of the synchronous gauge for the calculation of linear perturbations of SFDM models.

In Sec. 5, we will analyze the suppression of power in the mass PS and discuss the possibilities to constrain the model with current and future galaxy surveys. We will also discuss, from a qualitative point of view, the effect of the aforementioned suppression in the present mass function of halos. Finally, Sec. 6 will be devoted to a general discussion of the main results and conclusions.

\section{SFDM background dynamics}

For the background dynamics, we shall consider a spatially-flat Universe with line element $d s^{2}=-d t^{2}+a^{2}(t) \delta_{i j} d x^{i} d x^{j}$, and populated with standard matter fluids such as: radiation, neutrinos, baryons, and a cosmological constant $\Lambda$, together with dark matter described by a scalar field $\phi$ endowed with the quadratic potential (1.1); the corresponding Lagrangian density of the latter is $\mathcal{L}_{\phi}=-(1 / 2)(\partial \phi)^{2}-(1 / 2) m^{2} \phi^{2}$. We will refer to the model as $\Lambda$ SFDM, to emphasize the role of the scalar field as dark matter, with a cosmological constant $\Lambda$ still playing the role of dark energy. 


\subsection{Mathematical background}

If we use $\rho_{I}$ and $p_{I}$ to denote the energy density and pressure, respectively, for each matter fluid in the model apart from the scalar field, the full equations of motion of the $\Lambda$ SFDM model are:

$$
\begin{aligned}
H^{2} & =\frac{\kappa^{2}}{3}\left(\sum_{I} \rho_{I}+\rho_{\phi}\right) \\
\dot{H} & =-\frac{\kappa^{2}}{2}\left[\sum_{I}\left(\rho_{I}+p_{I}\right)+\left(\rho_{\phi}+p_{\phi}\right)\right], \\
\dot{\rho}_{I} & =-3 H\left(\rho_{I}+p_{I}\right), \\
\ddot{\phi} & =-3 H \dot{\phi}-m^{2} \phi
\end{aligned}
$$

where $\kappa^{2}=8 \pi G$, a dot denotes derivative with respect to cosmic time $t$, and $H$ is the Hubble parameter. The scalar field energy density $\rho_{\phi}$ and pressure $p_{\phi}$ are given by the known expressions:

$$
\rho_{\phi}=\frac{1}{2} \dot{\phi}^{2}+\frac{1}{2} m^{2} \phi^{2}, \quad p_{\phi}=\frac{1}{2} \dot{\phi}^{2}-\frac{1}{2} m^{2} \phi^{2} .
$$

In order to transform the Klein-Gordon (KG) equation (2.1d), we define a new set of variables[61]:

$$
x \equiv \frac{\kappa \dot{\phi}}{\sqrt{6} H}, \quad y \equiv-\frac{\kappa m \phi}{\sqrt{6} H}, \quad y_{1} \equiv 2 \frac{m}{H},
$$

In writing Eqs. (2.3), we are assuming that initially $\phi<0$ so that both variables $y, y_{1}$ start with positive values ${ }^{1}$. As a result, the KG equation can be written as a system of first order differential equations in the form:

$$
x^{\prime}=-\left(3+\frac{\dot{H}}{H^{2}}\right) x+\frac{1}{2} y y_{1}, \quad y^{\prime}=-\frac{\dot{H}}{H^{2}} y-\frac{1}{2} x y_{1}, \quad y_{1}^{\prime}=-\frac{\dot{H}}{H^{2}} y_{1},
$$

where a prime denotes derivative with respect to the number of $e$-foldings $N \equiv \ln \left(a / a_{i}\right)$, with $\left(a_{i}\right) a$ the (initial) scale factor of the Universe. (Hereafter, we label initial conditions with a sub-index $i$.)

We now apply a polar change of variables to the kinetic and potential variables in the form: $x=\Omega_{\phi}^{1 / 2} \sin (\theta / 2)$ and $y=\Omega_{\phi}^{1 / 2} \cos (\theta / 2)$, where $\Omega_{\phi} \equiv \kappa^{2} \rho_{\phi} / 3 H^{2}$ is the density parameter of the scalar field. This type of transformations was first proposed in[62] and recently applied for inflationary models in[63], but see also[64-66] for other similar definitions. Firstly, we rewrite the Friedman constraint (2.1a) and the acceleration equation (2.1b), respectively, as

$$
1=\sum_{j} \Omega_{j}+\Omega_{\phi}, \quad \frac{\dot{H}}{H^{2}}=-\frac{3}{2}\left(1+w_{t o t}\right),
$$

where

$$
\Omega_{I} \equiv \frac{\kappa^{2} \rho_{I}}{3 H^{2}}, \quad w_{t o t} \equiv \frac{p_{t o t}}{\rho_{t o t}}=\sum_{I} \Omega_{I} w_{I}+\Omega_{\phi} w_{\phi}
$$

\footnotetext{
${ }^{1}$ This means that the evolution of the scalar field $\phi$ starts on the left branch of the parabolic potential (1.1), which is not a limitation as the system of equations is invariant under the change $\phi \rightarrow-\phi$. To start the evolution on the right branch, the signs of $y, y_{1}$ just need to be changed.
} 
and $w_{I}=p_{I} / \rho_{I}$ is the barotropic equation of state (EoS) of the $I$-th matter fluid. Notice that we have defined the total EoS $w_{t o t}$ for the background dynamics in terms of the ratio of the total pressure $p_{t o t}$ to the total energy density $\rho_{t o t}$, whereas that of the scalar field is simply given by

$$
w_{\phi} \equiv \frac{p_{\phi}}{\rho_{\phi}}=\frac{x^{2}-y^{2}}{x^{2}+y^{2}}=-\cos \theta .
$$

That is, the new angular variable $\theta$ is directly related to the scalar field EoS.

After some straightforward algebra, the KG equation (2.1d) becomes:

$$
\begin{aligned}
\theta^{\prime} & =-3 \sin \theta+y_{1}, \\
y_{1}^{\prime} & =\frac{3}{2}\left(1+w_{t o t}\right) y_{1}, \\
\Omega_{\phi}^{\prime} & =3\left(w_{t o t}-w_{\phi}\right) \Omega_{\phi} .
\end{aligned}
$$

Eqs. (2.7) are a compact and neat representation of the KG equation, and they reveal that the true variables driving the scalar field dynamics are $\left(\theta, y_{1}, \Omega_{\phi}\right)$, with the field values $\phi$ and $\dot{\phi}$ now playing a secondary role. Eqs. (2.7) also show that the dynamics of the quadratic potential does not have any free physical parameters, as the successive transformations applied to the KG equation made the field mass $m$ become an implicit parameter in the final equations of motion ${ }^{2}$.

\subsection{Early, intermediate, and late time behaviors}

It is convenient to study the behavior of the scalar field under the new equations of motion. (2.7). For that we consider that there is a special time for the beginning of the scalar field oscillations around the minimum of the potential (1.1), that we will denote by its corresponding scale factor $a_{o s c}$. Equivalently, $a_{o s c}$ also corresponds to $\theta_{o s c}=\pi / 2$, which is the point at which the SFDM EoS first passes through zero, $w_{\phi}\left(\theta_{\text {osc }}\right)=0^{3}$.

As the field in our study will be part of the matter budget, we will also consider that the oscillations start before the time of radiation-matter equality at $a_{e q}$, and then we put the constraint $a_{o s c}<a_{e q}{ }^{4}$. Thus, for early times we will refer to the epoch of radiation domination (RD) before the onset of the scalar field oscillations, so that $a \ll a_{\text {osc }}$. Intermediate times will refer to $a<a_{o s c}$, still within the $\mathrm{RD}$, whereas late times will strictly refer to $a>a_{o s c}$. In terms of the angular variable $\theta$, the above times can be rephrased as: $0<\theta \ll \pi / 2$ for early times, $\theta<\pi / 2$ for intermediate times, and $\theta>\pi / 2$ for late times. This classification

\footnotetext{
${ }^{2}$ Strictly speaking, there is no need to consider $y_{1}$ as a separate variable, nor to take Eq. (2.7b) as an independent equation of motion. As shown in Eqs. (2.3), $y_{1}$ is just proportional to $H^{-1}$, and then only Eqs. (2.7a) and $(2.7 \mathrm{c})$ are enough to represent the KG equation (2.1d) when the scalar field potential is the quadratic one in Eq. (1.1). However, $y_{1}$ plays an independent role in cases with more involved scalar field potentials, and then we will consider it as such for reference in future works (see for instance[63] in the case of inflationary models). As we shall show, this approach will also be useful to find approximate solutions in Sec. 2.2 below.

${ }^{3}$ Usual treatments define $a_{\text {osc }}$ from the approximate relation $m \simeq 3 H\left(a_{\text {osc }}\right)$. Notice that our approach gives a more accurate definition of $a_{o s c}$ as it directly involves the scalar field EoS. As we shall see in Sec. 2.3, the exact value of $a_{o s c}$, which can be obtained from Eq. (2.13), is not necessary for the numerical calculations

4 This is an important assumption for the analytical expressions in this section. However, the same equations of motion can also be solved approximately for the case in which $a_{o s c}>a_{e q}$. One simple approach to do so is to find approximate solutions Of Eqs. (2.7) for $a<a_{e q}$ assuming radiation domination, and for $a>a_{e q}$ assuming matter domination. The two solutions would be matched together at $a=a_{o s c}$. This procedure would provide good enough expressions for purposes beyond those of the present paper.
} 
is in agreement with our assumption that at early times $\phi<0$, so that the scalar field starts its journey on the left branch of the quadratic potential (1.1). Moreover, that $\theta>0$ also implies, via Eqs. (2.3) and the polar change of variables, that initially $\dot{\phi}>0$, so that the field rolls down towards $\phi=0$.

Before proceeding further, we note that in our scheme the SFDM mass can be determined from the initial condition of the second potential variable as: $m=y_{1 i} H_{i} / 2$, see also Eqs. (2.3). In general terms, we can expect that at early enough times $m / H_{i} \ll 1$, which directly indicates also that $y_{1 i} \ll 1$. In terms of the original KG equation (2.1d), this implies that the scalar field dynamics is dominated by the friction term containing the Hubble parameter $H$, which then puts the field into a slow-roll phase with its energy density dominated by the potential term; this in turn translates into $x \ll y$, and then for the angular variable: $\theta_{i} \ll 1$. A similar result is expected for $\Omega_{\phi i} \ll 1$ if SFDM is to be subdominant during RD.

The above simple estimations indicate that the scalar field variables must have initial values very close to the following critical point of the dynamical system $(2.7):\left(\theta=0, y_{1}=\right.$ $0, \Omega_{\phi}=0$ ). Under this assumption, Eqs. (2.7) can be linearised around this critical point as:

$$
\theta^{\prime} \simeq-3 \theta+y_{1}, \quad y_{1}^{\prime} \simeq 2 y_{1}, \quad \Omega_{\phi}^{\prime} \simeq 4 \Omega_{\phi},
$$

where we have considered that $w_{t o t}=1 / 3$, as expected in $\mathrm{RD}$, and also that $w_{\phi} \simeq-1$ and $\sin \theta \simeq \theta$ for $\theta \ll 1$. The linear system (2.8) has the following solutions in terms of the scale factor $a$ :

$$
\begin{aligned}
\theta & =(1 / 5) y_{1}+C\left(a / a_{i}\right)^{-3}, \\
y_{1} & =y_{1 i}\left(a / a_{i}\right)^{2}, \\
\Omega_{\phi} & =\Omega_{\phi i}\left(a / a_{i}\right)^{4},
\end{aligned}
$$

with $C$ an integration constant. The angular variable $\theta$ has growing and decaying modes, and when the latter disappears we find that $5 \theta \simeq y_{1}$. We shall call this relation the attractor solution at early times between variables $\theta$ and $y_{1}$. As for the scalar field density parameter, its solution (2.9c) indicates that the SFDM energy density is constant during RD: $\rho_{\phi} \simeq$ $\Omega_{\phi} \rho_{r}=$ const $^{5}$.

For intermediate times, a more general solution of Eqs. (2.7a) and (2.7b) can be found perturbatively, as $\theta$ and $y_{1}$ remains reasonably small up to the time before the onset of oscillations at $\theta=\pi / 2$. Moreover, $\theta$ has a monotonic behavior, and then we can propose the ansatz:

$$
y_{1}(\theta)=\sum_{j=1} k_{j} \theta^{j}, \quad k_{j}=\text { const. }
$$

We would like to emphasize that $y_{1}(\theta)$ is all that is needed to find a full solution of the scalar field equations (2.1). Indeed, once with the values of $k_{j}$ at hand we can write Eq. (2.7a) as:

$$
\theta^{\prime}=\left(k_{1}-3\right) \theta+k_{2} \theta^{2}+\left(k_{3}+1 / 2\right) \theta^{3}+\ldots
$$

Eq. (2.11) can in principle be integrated at any desired order in $\theta$. For our purposes, it is enough to consider in Eq. (2.6) an expansion up to the third order, and then $y_{1}(\theta)=$ $k_{1} \theta+k_{2} \theta^{2}+k_{3} \theta^{3}$. The key step to find the coefficients $k_{j}$ is to write Eq. (2.7b) in the form

$$
y_{1}^{\prime}=\frac{d y_{1}}{d \theta} \theta^{\prime}=\frac{d y_{1}}{d \theta}\left(-3 \sin \theta+y_{1}\right)=2 y_{1},
$$

\footnotetext{
${ }^{5}$ As a side result, one can see that the attractor solutions implies that the evolution of the scalar field EoS at early times is approximately given by $w_{\phi} \simeq-1+\theta^{2} / 2=-1+(2 / 25) m^{2} / H^{2}$, which coincides at the lowest order with Eq. (A31) in[39]
} 
where we again assummed RD with $w_{t o t}=1 / 3$ for the last term on the rhs. After expanding both sides of Eq. (2.12) in powers of $\theta$, the resultant polynomials must have the same coefficients, and from this condition we obtain $k_{1}=5, k_{2}=0$, and $k_{3}=-5 / 18$. Finally, Eq. (2.11) can be integrated to find:

$$
\frac{\theta^{2}}{\theta_{i}^{2}}\left(\frac{9+\theta_{i}^{2}}{9+\theta^{2}}\right)=\left(a / a_{i}\right)^{4},
$$

which could be easily inverted to find $\theta=\theta(a)$ for $a<a_{\text {osc }}$. If necessary, we can also calculate $^{6} y_{1}=5 \theta-5 \theta^{3} / 18$, whereas $\Omega_{\phi}$ would still be given by Eq. (2.9c).

At late times $a>a_{o s c}$, the scalar field field starts to oscillate rapidly around the minimum of the potential with a decreasing amplitude, and then we expect $y_{1}$ to grow very quickly and to become the dominant term in Eq. (2.7a): $\theta^{\prime} \simeq y_{1}$. Changing back the derivative in the latter equation to the cosmic time $t$, we find that:

$$
\dot{\theta} \simeq 2 m \rightarrow \theta \simeq 2 m t, \text { and } w_{\phi} \simeq-\cos (2 m t) .
$$

Irrespective of the domination era after the onset of oscillations, we can see that the SFDM EoS will oscillate around zero with a fixed frequency, in terms of the cosmic time, given by the field mass $m$. The oscillations happen in a time scale much smaller than that of the expansion rate of the Universe, which means that $H^{-1}>m^{-1}$. The Hubble parameter is a decreasing function of time, and we can only expect that $y_{1}=2 m / H \gg 1$ as the Universe continues its expansion for $a>a_{\text {osc }}$.

For this reason, it is generically considered that the SFDM energy density evolves, on average, with a matter-like EoS: $\left\langle w_{\phi}\right\rangle \simeq 0[36,37]$. This expectation has also been confirmed by accurate numerical simulations[14, 15, 17, 32] (see also Fig. 11 in the Appendix A), and then we can safely take for granted that the SFDM background density will evolve as a matter component, $\rho_{\phi} \propto a^{-3}$, irrespectively of the presence of other fluid components.

\subsection{Initial conditions}

In order to find numerical solutions of the dynamical system (2.7), all that remains is to find appropriate initial conditions $\left(\theta_{i}, y_{1 i}, \Omega_{\phi i}\right)$, so that we can get the desired SFDM contribution $\Omega_{\phi 0}$ at the present time. This is done in most CMB codes, in terms of the standard field scheme, by the implementation of a shooting procedure to determine the correct initial values $\phi_{i}$ and $\dot{\phi}_{i}[60]$. However, it is still necessary to find a guess value, commonly obtained trough a cumbersome calculation[18, 39], to initiate the shooting method. As we shall show below, there is a more direct path to find the correct initial conditions in terms of our new dynamical variables.

We start by writing down an equation for the initial value of the SFDM density parameter $\Omega_{\phi i}$. For that, we first recall that once the scalar field starts to oscillate rapidly, its energy density redshifts as $a^{-3}$, so that its ratio with respect to the radiation component is: $\Omega_{\phi} / \Omega_{r}=\left(\Omega_{\phi 0} / \Omega_{r 0}\right) a$ for $a>a_{o s c}$. In particular, we can write $\Omega_{\phi o s c} \simeq a_{o s c} \Omega_{r o s c}\left(\Omega_{\phi 0} / \Omega_{r 0}\right) \simeq$ $a_{o s c}\left(\Omega_{\phi 0} / \Omega_{r 0}\right)$, where the last expression takes into account that $\Omega_{\text {rosc }}=1$ if the Universe is still dominated by radiation at $a=a_{\text {osc }}$. From Eq. (2.9c), we also know that $\Omega_{\phi o s c} \simeq\left(a_{o s c} / a_{i}\right)^{4} \Omega_{\phi i}$, and all together with Eq. (2.13) we finally find that:

$$
\Omega_{\phi i} \simeq a_{i} \frac{\Omega_{\phi 0}}{\Omega_{r 0}}\left(\frac{a_{i}}{a_{o s c}}\right)^{3}=a_{i} \frac{\Omega_{\phi 0}}{\Omega_{r 0}}\left[\frac{4 \theta_{i}^{2}}{\pi^{2}}\left(\frac{9+\pi^{2} / 4}{9+\theta_{i}^{2}}\right)\right]^{3 / 4} .
$$

\footnotetext{
${ }^{6}$ Notice that according to this solution the ratio of the SFDM mass to the Hubble parameter at the start of the oscillations is $m / H_{o s c} \simeq 3.39$.
} 
The search for good initial conditions is now quite simple. We first need to set up by hand the desired contributions of the radiation and the SFDM components at the present time: $\Omega_{r 0}, \Omega_{\phi 0}$, together with the initial value of the scale factor $a_{i}$. Then, we choose an initial value $\theta_{i}$, use the attractor solution of Eq. (2.9a) at early times to find: $y_{1 i}=5 \theta_{i}$, and finally take Eq. (2.15) to find the corresponding initial value $\Omega_{\phi i}$.

To conclude this section, it must be said that the ratio of the SFDM mass to the initial Hubble parameter is a derived value calculated from the initial conditions: in terms of the attractor relation for the potential variable $y_{1}$, we find that $m / H_{i}=y_{1 i} / 2=(5 / 2) \theta_{i}$. A more useful formula can be written in terms of the present Hubble parameter $H_{0}$ as:

$$
\frac{m}{H_{0}}=\frac{H_{i}}{H_{0}} \frac{y_{1 i}}{2}=\frac{5}{2} \Omega_{r 0}^{1 / 2} a_{i}^{-2} \theta_{i},
$$

where we have again assumed RD in the form $H_{i}^{2}=\left(\kappa^{2} / 3\right) \rho_{r 0} a_{i}^{-4}$ to write the last expression on the rhs. Eq. (2.16) shows again the interplay of the initial conditions to set the value of the scalar field mass: everything else the same, different masses $m$ are just obtained by taking different values of the initial condition $\theta_{i}$.

\subsection{Modifications in CLASS}

The scheme described above to evolve the scalar field equations of motion was incorporated in an amended version of the freely-available code CLASS $[60,67,68]^{7}$. In order to be consisten with the background evolution of other matter components within CLASS, the scalar field energy density and pressure were calculated, respectively, from the expressions:

$$
\rho_{\phi}=\frac{\Omega_{\phi}}{1-\Omega_{\phi}}\left(\rho_{r}+\rho_{m}+\rho_{\Lambda}\right), \quad p_{\phi}=-\cos \theta \cdot \rho_{\phi} .
$$

The above formulae are found under the assumption that $\Omega_{\phi}=\rho_{\phi} / \rho_{t o t}$, where $\rho_{t o t}$ is the total energy density at any given time. This assumption is in agreement with a spatially flat Universe, for which the total energy density coincides with the critical one $\rho_{\text {tot }}=\rho_{\text {crit }}=$ $3 H^{2} / 8 \pi G$. Notice that our scheme does not exclude the presence of other dark matter components (like CDM) apart from SFDM. Actually, following the given structure within CLASS, all actual matter contributions are put in by hand, except for that of SFDM, which is calculated the last from the fulfillment of the Friedman constraint for a spatially flat Universe (which is also the scheme within CLASS to deal with quintessence fields). Then, the amended code can also be used to study mixed dark matter models (as in[39]), and no change for such a case would required in the formalism presented throughout this work. However, for our present purposes, we shall consider that SFDM is the only dark matter component.

Even though Eqs. (2.7) are more convenient for accurate numerical solutions than the traditional field approach, they still require a highly efficient solver of differential equations to deal properly with the numerical stiffness of the scalar field oscillations. In order to circumvent this difficulty, we implemented a cut-off procedure on the trigonometric functions in the form:

$$
\left\{\cos _{\star} \gamma, \sin _{\star} \gamma\right\} \equiv(1 / 2)\left[1-\tanh \left(\gamma^{2}-\gamma_{\star}^{2}\right)\right]\{\cos \gamma, \sin \gamma\},
$$

\footnotetext{
${ }^{7}$ It must be noticed that the equations of motion (2.7) are written with derivatives with respect to the number of $e$-foldings $N$. The derivatives can be changed to conformal time $\tau$ (the preferred time in CMB codes) via the well-known transformation $(d / d \tau)=a H(d / d N)=\mathcal{H}(d / d N)$, where $\mathcal{H}$ is the Hubble parameter itself given in terms of the conformal time
} 
and then $\left\{\cos _{\star} \gamma, \sin _{\star} \gamma\right\} \rightarrow 0$ for $\gamma>\gamma_{\star}$. The threshold value $\gamma_{\star}$ was chosen empirically to neglect the trigonometric functions everywhere once the equation solver in CLASS was no longer able to keep an accurate track of the rapid oscillations of the scalar field. After some experimentation, we found that a convenient value is $\gamma_{\star}=10^{2}$.

An illustrative physical example is the scalar field EoS, which was written inside CLASS as: $w_{\phi}=-\cos _{\star} \theta$. As we saw in Sec. 2.2, during the phase of rapid oscillations $\theta \simeq y_{1} / 2 \simeq$ $m / H$, and then in this case the threshold value $\gamma_{\star}$ means $\theta_{\star} \simeq m / H_{\star} \simeq 10^{2}$. In other words, $w_{\phi}$ is set to zero once the time scale for the expansion of the Universe is two orders of magnitude larger than the period of one oscillation in the EoS, irrespective of the value of the considered SFDM mass $m$. As explained in more detail in the Appendix A, the implemented numerical cut-off (2.18) worked well, and did not introduce spurious effects into the numerical solutions.

As for the initial conditions, it must be said that Eq. (2.15) is not quite the correct initial condition for the SFDM density parameter, and we still needed to finely tune $\Omega_{\phi i}$ at the beginning of every numerical run. For that, we wrote $\Omega_{\phi i}=A \times$ Eq. (2.15), where the value of $A$ was adjusted with the shooting method already implemented within CLASS for scalar field models. A few iterations of the shooting routine were always enough to find the correct $\Omega_{\phi i}$ that leads to the desired $\Omega_{\phi 0}$ with a very high precision; in all instances it was found that $A=\mathcal{O}(1)$.
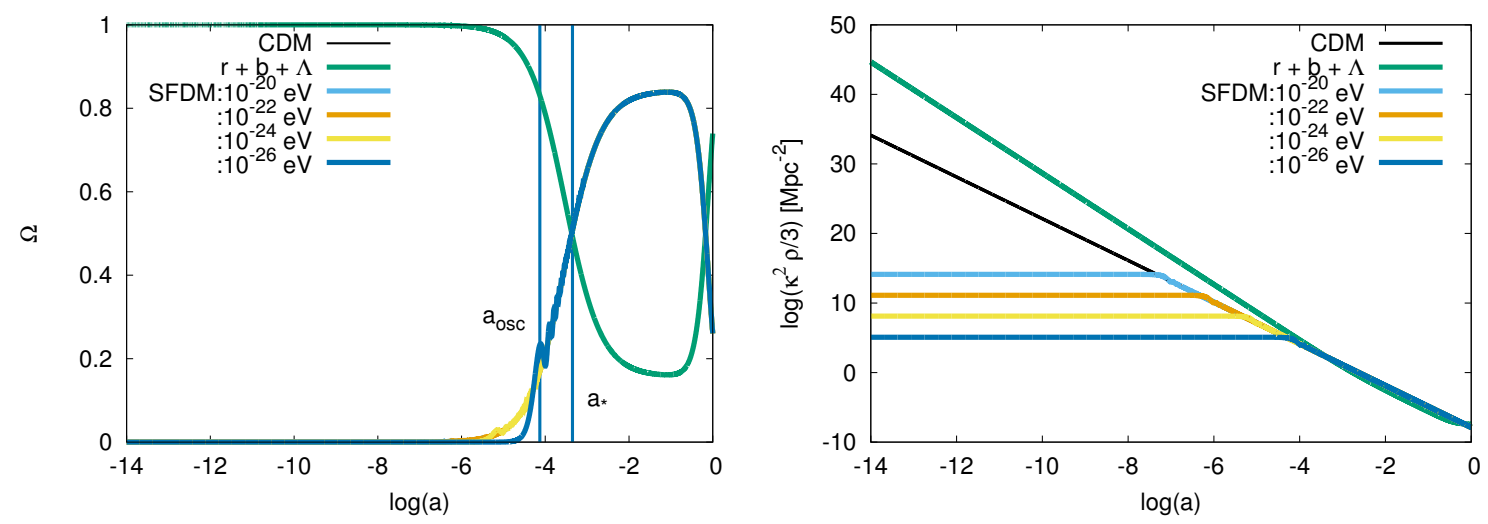

Figure 1. Numerical solutions of the background equations (2.1) and (2.7) with a scalar field as dark matter at late times. The label CDM in the plots refers to the standard dark matter component, whereas $r, b$ and $\Lambda$ stand for radiation (photons and neutrinos), baryons, and a cosmological constant. Shown are: (Left) the density parameters $\Omega$ (see Eqs. (2.5)), and (Rigth) the energy densities $\rho$, of the different matter components. Notice that the standard evolution of the $\Lambda$ CDM model is fully recovered at late times. The onset of the field oscillations is manifest in the wiggles present in the curves, although the most noticeable cases correspond to the lightest masses, for which the oscillations start closely to the time of radiation-matter equality at $a \simeq 10^{-4}$. As an example, the vertical lines indicate the start of the oscillations at $a_{o s c}$, and the application of the cut-off for rapid oscillations at $a_{*}$, for the lightest case of $m=10^{-26} \mathrm{eV}$. See Secs. 2.2, 2.4, and the Appendix A.1, for more details.

\subsection{Numerical results for the background}

Some numerical results from CLASS of background quantities are shown in Figs. 1, for a Universe in which the scalar field is the only dark matter component, that is, $\Omega_{\phi 0}=\Omega_{C D M 0}$, whereas all other matter components (radiation, baryons, and $\Lambda$ ) are the same as reported 
by the Planck collaboration for the concordance model $\Lambda$ CDM[69]. The mass of the SFDM particle in the given examples is indicated in the plots, and in each case was calculated from the initial condition $\theta_{i}$ through Eq. (2.16).

A general feature in all the numerical examples is that the SFDM energy density $\rho_{\phi}$ is constant at very early times, when its dynamics is dominated by its potential energy. Once the oscillations start, the energy density scales as a matter component, and the evolution of the density parameters clearly show that the subsequent epochs of matter and $\Lambda$ domination are indistinguishable from those of $\Lambda \mathrm{CDM}$. The onset of oscillations can be clearly seen in the evolution of the density parameter $\Omega_{\phi}$, specially in the cases with the lightest masses. Actually, the oscillations start well before the time of radiation-matter equality except for the lightest case $m \simeq 10^{-26} \mathrm{eV}$. A detailed description of the solution of $\Omega_{\phi}$ can be found in the Appendix A, where we also show that the cut-off (2.18) helps CLASS to calculate effortlessly the right solution at late times.

\section{SFDM linear perturbations}

The linear perturbations of scalar fields, and its influence in the CMB and other related observables, have been studied intensively in the specialized literature under a variety of different circumstances, see for instance[15, 18, 21, 22, 32, 39, 70-74] and references therein. Here, we aim to present a new study of field perturbations that, in many aspects, mirrors the one we presented for the background in Sec. 2.

Following common wisdom, here we shall assume the synchronous gauge of metric perturbations, with the line element $d s^{2}=-d t^{2}+a^{2}(t)\left(\delta_{i j}+h_{i j}\right) d x^{i} d x^{j}$. Notice that we will not work with the standard conformal time $\tau$, and then a dot still means derivative with respect to the cosmic time $t$. The scalar field is given by $\phi(x, t)=\phi(t)+\varphi(x, t)$, where $\phi(t)$ is the background (homogeneous) field, and $\varphi$ is its linear perturbation. Thus, the linearized KG equation for a Fourier mode $\varphi(k, t)$ reads[37, 70-72]:

$$
\ddot{\varphi}=-3 H \dot{\varphi}-\left(k^{2} / a^{2}+m^{2}\right) \varphi-\frac{1}{2} \dot{\phi} \dot{h},
$$

where $h$ is the trace of scalar metric perturbations (with $\dot{h}$ known as the metric continuity), and $k$ is a comoving wavenumber. The perturbations in density $\delta \rho_{\phi}$, pressure $\delta p_{\phi}$, and velocity divergence $\Theta_{\phi}$, are given, respectively, by[71, 72, 75]:

$$
\begin{aligned}
\delta \rho_{\phi} & =\dot{\phi} \dot{\varphi}+\partial_{\phi} V \varphi, \\
\delta p_{\phi} & =\dot{\phi} \dot{\varphi}-\partial_{\phi} V \varphi, \\
\left(\rho_{\phi}+p_{\phi}\right) \Theta_{\phi} & =\left(k^{2} / a\right) \dot{\phi} \varphi .
\end{aligned}
$$

The standard field approach for scalar field perturbations is to solve Eq. (3.1) for a given background evolution to find $\varphi$ and $\dot{\varphi}$, which are then used to calculate the perturbative quantities (3.2). At a heuristic level, and for a rapidly oscillating scalar field, we can see that its linear perturbations grow if the fields in the KG equations (2.1c) and (3.1) have similar natural frequencies, which would happen for scales such that $k \ll m$, whereas in the opposite case $k \gg m$, we expect a suppression of the perturbations.

The true behavior of scalar field perturbations is actually more complex, and numerical solutions are unavoidable. However, Eq. (3.1) suffers from the same stiffness problem of the background equations, mainly because of the rapidly oscillatory terms that arise on the 
rhs from the presence of $m^{2}$ and $\dot{\phi}$, which also renders it impractical for numerical use in CMB codes[15, 18, 39]. An alternative, and second approach to the study of scalar field perturbations, is to transform Eq. (3.1) into an equivalent set of fluid equations, as was first done in the so-called formalism of generalized dark matter[75]. Such transformation is still impractical for numerical purposes (as already noted in[75]) in the case of rapidly oscillating scalar fields, mostly because some fluid terms, like the sound speed $c_{s}^{2}=\delta p_{\phi} / \delta \rho_{\phi}$, are ill behaved during the oscillating phase of the scalar field.

Such difficulties in the fluid approximation have not deterred its ample use in the specialized literature[18, 21, 22, 29, 39, 73, 74]. One common corrective step in this method, that was firstly suggested in[37], is to assume an approximate ansatz for the field oscillations, and then to derive from it averaged fluid equations freed from any troublesome behavior[21, 22, 39]. Although highly popular, this approach requires some intrusive pre-handling of the equations of motion that obscures the physical mechanisms at work in the evolution of SFDM perturbations.

We propose a third approach, based in a new field transformation of Eq. (3.1) quite similar to that we used for the background variables:

$$
\begin{aligned}
& u=\sqrt{\frac{2}{3}} \frac{\kappa \dot{\varphi}}{H}=-\Omega_{\phi}^{1 / 2} e^{\alpha} \cos (\vartheta / 2), \\
& v=\sqrt{\frac{2}{3}} \frac{\kappa m \varphi}{H}=-\Omega_{\phi}^{1 / 2} e^{\alpha} \sin (\vartheta / 2) .
\end{aligned}
$$

For a simple interpretation of the new variables $\vartheta$ and $\alpha$, it is useful to define the density $\delta_{\phi} \equiv$ $\delta \rho_{\phi} / \rho_{\phi}$, and pressure $\delta_{p_{\phi}} \equiv \delta p_{\phi} / \rho_{\phi}$ contrasts, respectively, from the physical quantities (3.2). In summary, we find:

$$
\begin{aligned}
\delta_{\phi} & =-e^{\alpha} \sin (\theta / 2-\vartheta / 2), \\
\delta_{p_{\phi}} & =-e^{\alpha} \sin (\theta / 2+\vartheta / 2), \\
\left(\rho_{\phi}+p_{\phi}\right) \Theta_{\phi} & =-\frac{k^{2}}{a m} \rho_{\phi} e^{\alpha} \sin (\theta / 2) \sin (\vartheta / 2) .
\end{aligned}
$$

The appearance of oscillations in the perturbative quantities can be easily spotted in Eqs. (3.4); they result from the combination of the background and perturbative angular variables $\theta$ and $\vartheta$. Moreover, we can see that $e^{\alpha}$ can be simply interpreted as the amplitude of the scalar field density and pressure contrasts.

After long, but otherwise straightforward, calculations, we find that the equations of motion of the new variables, as derived from the perturbed KG equation (3.1), are:

$$
\begin{aligned}
\vartheta^{\prime} & =3 \sin \vartheta+y_{1}+2 \omega \sin ^{2}(\vartheta / 2)-2 e^{-\alpha} h^{\prime} \sin (\theta / 2) \sin (\vartheta / 2), \\
\alpha^{\prime} & =-\frac{3}{2}(\cos \vartheta+\cos \theta)-\frac{\omega}{2} \sin \vartheta+e^{-\alpha} h^{\prime} \sin (\theta / 2) \cos (\vartheta / 2) .
\end{aligned}
$$

There are two remarkable features in Eqs. (3.5). The first one is that the Hubble parameter $H$ does not appear explicitly, and then the only connection of the perturbation variables to the background expansion is through the presence of $\theta$ and $y_{1}$. The second feature is that $y_{1}$, in a similar way as in Eq. (2.7a), should at some point become the dominant term in Eq. (3.5a), and then we must expect that at late times $\vartheta \simeq \theta=2 m t$. Hence, the perturbation variables $\varphi$ and $\dot{\varphi}$, see Eqs. (3.3), will also be rapidly oscillating functions just as their background counterparts. 
Interestingly enough, Eqs. (3.5) quite naturally indicate the presence of a characteristic wavenumber in scalar field perturbations. For simplicity, we have denoted the ratio $\omega=$ $k^{2} / k_{J}^{2}$, where

$$
k_{J} \equiv a \sqrt{m H},
$$

Notice that $k_{J}$ is a time-dependent quantity that has been previously identified, sometimes just heuristically, as the comoving Jeans wavenumber of linear perturbations in scalar field models, see $[14,18,20,39,75,76]$ and references therein. Its present, and then physical, value is:

$$
\frac{k_{J 0}}{\mathrm{Mpc}^{-1}}=0.68\left(\frac{m}{10^{-23} \mathrm{eV}}\right)^{1 / 2}\left(\frac{H_{0}}{\mathrm{~km} \mathrm{~s}^{-1} \mathrm{Mpc}^{-1}}\right)^{1 / 2} .
$$

Actually, if the initial conditions are given in $\mathrm{RD}$, we find that the variation of the Jeans wavenumber is just proportional to the present density parameter of radiation: $k_{J i} / k_{J 0}=$ $a_{i} \sqrt{H_{i} / H_{0}}=\Omega_{r 0}^{1 / 4}$. This also means that in a typical cosmological evolution $k^{2} / k_{J}^{2} \lesssim 1$ for most of the scales of interest in structure formation, except in the case of extremely light masses $m \ll 10^{-23} \mathrm{eV}$. A complete study of the effects of the Jeans wave number $k_{J}$ in the evolution of SFDM perturbations will be presented in Sec. 4 below.

\subsection{Early time behavior}

As for the background case, it is convenient to make a description of the early time behavior of the scalar field perturbations from the point of view that Eqs. (3.5) is a dynamical system, with at least one meaningful critical point as $a \rightarrow 0(N \rightarrow-\infty)$, which will then act as the source point for the physical solutions. In contrast to the background equations (2.7), the dynamical system (3.5) is not autonomous due to the presence of the background quantities $\theta$ and $y_{1}$, and the metric term $h^{\prime}$, which together act as an external time-dependent force term for the perturbations.

If we assume RD, then we know from Eqs. (2.9) that the behavior of the background variables in terms of the scale factor is: $5 \theta=y_{1}=y_{i}\left(a / a_{i}\right)^{2}$, whereas that of the metric perturbation must be $h=h_{i}\left(a / a_{i}\right)^{2}$, where $h_{i}$ is a constant. This last expression comes from the well known solutions of linear perturbations in a RD universe with adiabatic initial conditions, see for instance[39, 72] for more details. In order to find their critical point, we rewrite Eqs. (3.5) in the form:

$$
\begin{aligned}
\vartheta^{\prime} & =2 \sin (\vartheta / 2)\left[3 \cos (\vartheta / 2)+\omega \sin (\vartheta / 2)-e^{-\alpha} h^{\prime} \sin (\theta / 2)\right]+y_{1}, \\
\alpha^{\prime} & =-\cos (\vartheta / 2)\left[3 \cos (\vartheta / 2)+\omega \sin (\vartheta / 2)-e^{-\alpha} h^{\prime} \sin (\theta / 2)\right]+3 \sin ^{2}(\theta / 2) .
\end{aligned}
$$

By simple inspection in Eq. (3.7a), we see that one critical point as $a \rightarrow 0$ is $\vartheta=0$. If we substitute this value into Eq. (3.7b) we find that:

$$
\alpha^{\prime} \simeq-3+e^{-\alpha} h^{\prime} \sin (\theta / 2)+3 \sin ^{2}(\theta / 2) .
$$

If we drop the last term in Eq. (3.7c) for being of a higher order in the expansion of $\theta$ (which is a small quantity), we find that a first integral is: $e^{\alpha} \simeq(2 / 7) h \sin (\theta / 2)$. Whereas $\vartheta$ reaches a true critical value, the critical condition on $\alpha$ just indicates its asymptotic behavior close to the point $a=0: \alpha \sim 4 N^{8}$.

\footnotetext{
${ }^{8}$ In this respect, we cannot find a true critical point in the phase space spanned by the variables $(\vartheta, \alpha)$. However, the critical point exists, at least formally, if the dynamical variables are $\left(\vartheta, e^{\alpha}\right)$, and would correspond to $\left(\vartheta=0, e^{\alpha} \rightarrow 0\right)$.
} 
We can now calculate the solution of $\vartheta$ to the next order close to the critical point. Considering a perturbation of the form $\vartheta=0+\delta \vartheta$, then we find that at the lowest order Eq. (3.7a) becomes: $\delta \vartheta^{\prime}=-4 \delta \vartheta+y_{1}$, from which we readily obtain that the growing solutions is $\delta \vartheta=(1 / 6) y_{1}=(5 / 6) \theta$. Therefore, the early-time solutions of the scalar field perturbations are represented by the expressions:

$$
\vartheta=(5 / 6) \theta, \quad e^{\alpha}=(1 / 7) h \theta .
$$

Eqs. (3.8) can be considered the attractor solutions of SFDM perturbations in the very early universe, and from them we can extract the right initial conditions for the scalar field perturbations. One important property of Eqs. (3.8) is that they do not depend on the wavenumber $k$, and then they are of general applicability for all scales.

Notice also that Eqs. (3.8) mean that the behavior of the scalar field density contrast close to the critical point, from Eq. (3.4a), can be cast in the form

$$
\delta_{\phi} \simeq-\frac{1}{21} h \sin ^{2}(\theta / 2)=-\frac{1}{42} h\left(1+w_{\phi}\right),
$$

and then the adiabatic condition $\delta_{\phi}=0$ is accomplished as the scalar field EoS $w_{\phi} \rightarrow-1$ (see Eq. (2.6)) for $a \rightarrow 0[39,72]$. Likewise, we can calculate the asymptotic expressions for the pressure contrast and the velocity divergence; from Eqs. (3.4b) and (3.4c) we find, respectively, that:

$$
\begin{aligned}
\delta_{p_{\phi}} & \simeq-\frac{11}{21} h \sin ^{2}(\theta / 2)=-\frac{11}{42} h\left(1+w_{\phi}\right), \\
\left(\rho_{\phi}+p_{\phi}\right) \Theta_{\phi} & \simeq-\frac{5}{21} \frac{k^{2}}{a m} \rho_{\phi} h \sin ^{3}(\theta / 2) .
\end{aligned}
$$

We see that both quantities also vanish as $a \rightarrow 0$.

\subsection{Gauge ambiguities and matter perturbations}

One of the favourite gauges for the numerical evolution of perturbations is the synchronouns gauge, mostly for its reliability and straightforward formulation for different matter components. It is known, however, that this gauge can only be completely determined if additional constraints are taken into account. The standard procedure is to fix its remaining degree of freedom by considering that all calculations are performed in the comoving frame of the cold dark matter component, in which the corresponding velocity perturbation vanishes, i.e. $\Theta_{\mathrm{CDM}}=0[77]$. It is clear that one must then be concerned about this gauge fixing in the cases where a scalar field is the dark matter, and specially if it is the only such component[78].

Most papers in the literature assume, under the fluid approximation of the equations of motion, that a rest framework can be established for SFDM much in the same way as for $\mathrm{CDM}$; the physical condition that must be satisfied is

$$
\left(\rho_{\phi}+p_{\phi}\right) \Theta_{\phi}=0 .
$$

This certainly can be done once the scalar field is oscillating and redshifting as matter[18, 21, $22,39]$, but the concern about the gauge issue remains if one wishes to start a cosmological evolution well before the onset of the field oscillations. Although it is possible to consider the Newtonian gauge[32], or better a gauge-invariant evolution[41], we adopted the practical point of view that all calculations are made in a frame comoving with a pressureless dust 
component. This is the standard assumption in CLASS, and actually we still needed to consider a tiny contribution of $\mathrm{CDM}\left(\Omega_{\mathrm{CDM} 0}<10^{-6}\right)$ so that CLASS would be able to run satisfactorily.

In this sense, because we are not working in the frame comoving with the scalar field, we should not expect the accomplishment of Eq. (3.10), but rather, as we shall see in Secs. 4.3 and 4.4 below, the scalar field perturbations must behave as those of a pressureless component. Such behavior is particularly clear in the case of the velocity perturbations, as the quantity $\left(\rho_{\phi}+p_{\phi}\right) \Theta_{\phi}$ evolves quite similarly to that of baryons, see Sec. 4.2 and Fig. 6.

Finally, there is some concern also about the inclusion of SFDM perturbations into the matter spectrum, see for instance the discussion in Sec. IV B in[39]. Here we will adopt a conservative point of view, also implicitly assumed within the perturbations module of CLASS, that the term matter perturbation comprises the perturbations coming from all components behaving as CDM at late times. This criterion is certainly fulfilled by the SFDM models studied here, as they are all able to reach a cold matter behavior before the present time. Hence, in the definition of the matter spectrum we just write $\delta_{m}=$ $\left(\delta \rho_{b}+\delta \rho_{\phi}\right) /\left(\rho_{b}+\rho_{\phi}\right)$ without making any distinction about the time before or after the onset of the field oscillations. One consequence of this is that the matter spectrum is mainly determined by the baryon perturbations at early times, but by the SFDM perturbations at late times once the scalar field dominates the matter sector.

\subsection{Modifications in CLASS}

The numerical evolution of the scalar field perturbations within CLASS is trickier than the evolution of the background, mainly because we need to take care of the different time scales that explicitly appear in the equations of motion, represented here by the angular variables $\theta$, $\vartheta$, and their combinations $\theta \pm \vartheta$. In order to make CLASS keep a good track of the evolution, it is convenient to use the following equations of motion that result from the combination of Eqs. (2.7a) and (3.5a):

$$
\begin{aligned}
& \tilde{\vartheta}^{\prime}=-3[\sin \theta+\sin (\theta-\tilde{\vartheta})]-[1-\cos (\theta-\tilde{\vartheta})] \omega+e^{-\alpha} h^{\prime}[\cos (\tilde{\vartheta} / 2)-\cos (\theta-\tilde{\vartheta} / 2 \nmid(3.11 \mathrm{a}) \\
& \alpha^{\prime}=-\frac{3}{2}[\cos (\theta-\tilde{\vartheta})+\cos \theta]-\frac{\omega}{2} \sin (\theta-\tilde{\vartheta})+\frac{1}{2} e^{-\alpha} h^{\prime}[\sin (\tilde{\vartheta} / 2)+\sin (\theta-\tilde{\vartheta} / 2)],(3.11 \mathrm{~b})
\end{aligned}
$$

where we have defined the new angular variable: $\tilde{\vartheta}=\theta-\vartheta$. As anticipated in Sec. 3, variables $\theta$ and $\vartheta$ will always have a very similar evolution, and then variable $\tilde{\vartheta}$, whose equation of motion (3.11a) does not contain $y_{1}$, will just measure the small difference between the two. Eqs. (3.11) together are the fundamental set of equations of motion for scalar field perturbations that were implemented in CLASS. The initial conditions for the new angular variables $(\tilde{\vartheta}, \alpha)$ are inferred from the attractor conditions (3.8), and then:

$$
\tilde{\vartheta}_{i}=(1 / 6) \theta_{i}, \quad \alpha_{i}=\ln \left(h_{i} \theta_{i} / 7\right) .
$$

In order to deal with the rapidly oscillating terms in the perturbation equations, we also applied to them the cut-off procedure of Eq. (2.18), and then we made the replacements $\{\cos , \sin \} \rightarrow\left\{\cos _{\star}, \sin _{\star}\right\}$ for all the trigonometric functions in Eqs. (3.11) containing $\theta$ in the argument. Like in the case of the background variables, those trigonometric functions were numerically neglected once their corresponding arguments reached a high-frequency regime. For instance, the second term on the rhs of Eq. (3.11a) was written as $\sin _{\star}(\theta-\tilde{\vartheta})$, and then the cut-off to its evolution was applied once $\theta-\tilde{\vartheta}>10^{2}$. Being $\theta$ the dominant angular 
variable, this also means that the rapidly oscillating terms are all cut-off at the same time for both the background and the perturbed equations of motion.

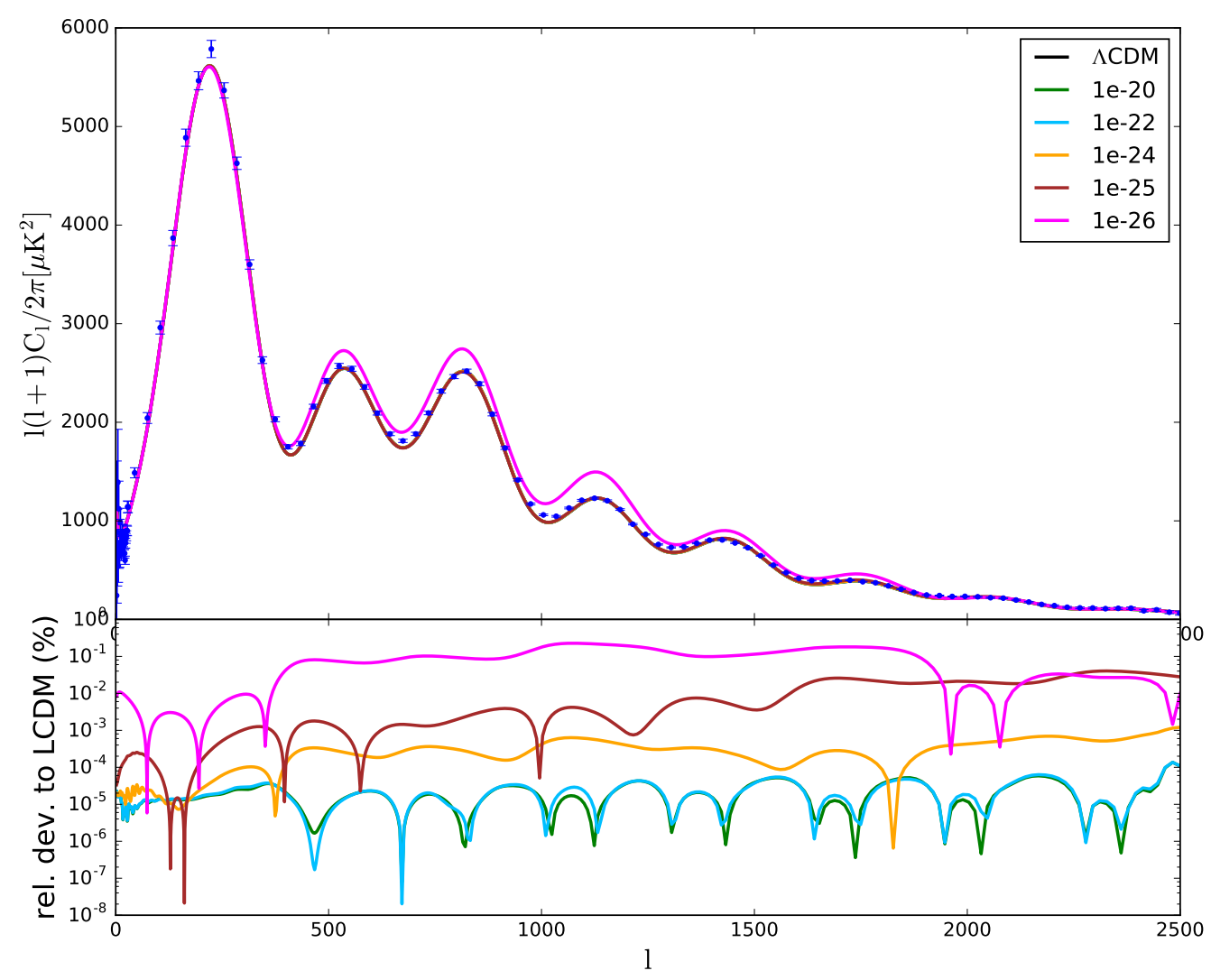

Figure 2. CMB temperature spectra for $\Lambda \mathrm{SFDM}$, as compared to the standard $\Lambda \mathrm{CDM}$ case. The differences are marginal for masses $m \gtrsim 10^{-24} \mathrm{eV}$, but they become quite noticeable for the lightest cases. Blue dots correspond to the Planck 2015 observations for binned high-l and low-l [79]

The equations of motion of perturbations were coded in CLASS following the formalism of the synchronous gauge, which is permitted as long as CDM is part of the matter budget. As mentioned before in Sec. 3.2, our simulations considered that $\Omega_{C D M 0}=10^{-6}$, so that CLASS could still be able to fix the gauge completely. It must be remarked here that all SFDM physical quantities were written inside CLASS exactly as presented in the text, without any handling of them except for the cut-off of those trigonometric terms that were able to reach a high-frequency regime. For example, as part of the source terms in the perturbed Einstein equations, the SFDM density contrast was written exactly as $\delta_{\phi}=-e^{\alpha} \sin (\tilde{\vartheta} / 2)$, whereas the pressure contrast was written as $\delta_{p_{\phi}}=-e^{\alpha} \sin _{\star}(\theta+\tilde{\vartheta} / 2)$.

In Figs. 2 and 3 we show the results from CLASS corresponding to the CMB temperature and mass power spectra, for the same range of masses as in the background dynamics: $10^{-26}<\mathrm{m} / \mathrm{eV}<10^{-20}$, and compare them with the standard $\Lambda \mathrm{CDM}$ model. In general terms, SFDM perturbations resemble very much those of the standard CDM plots, except 


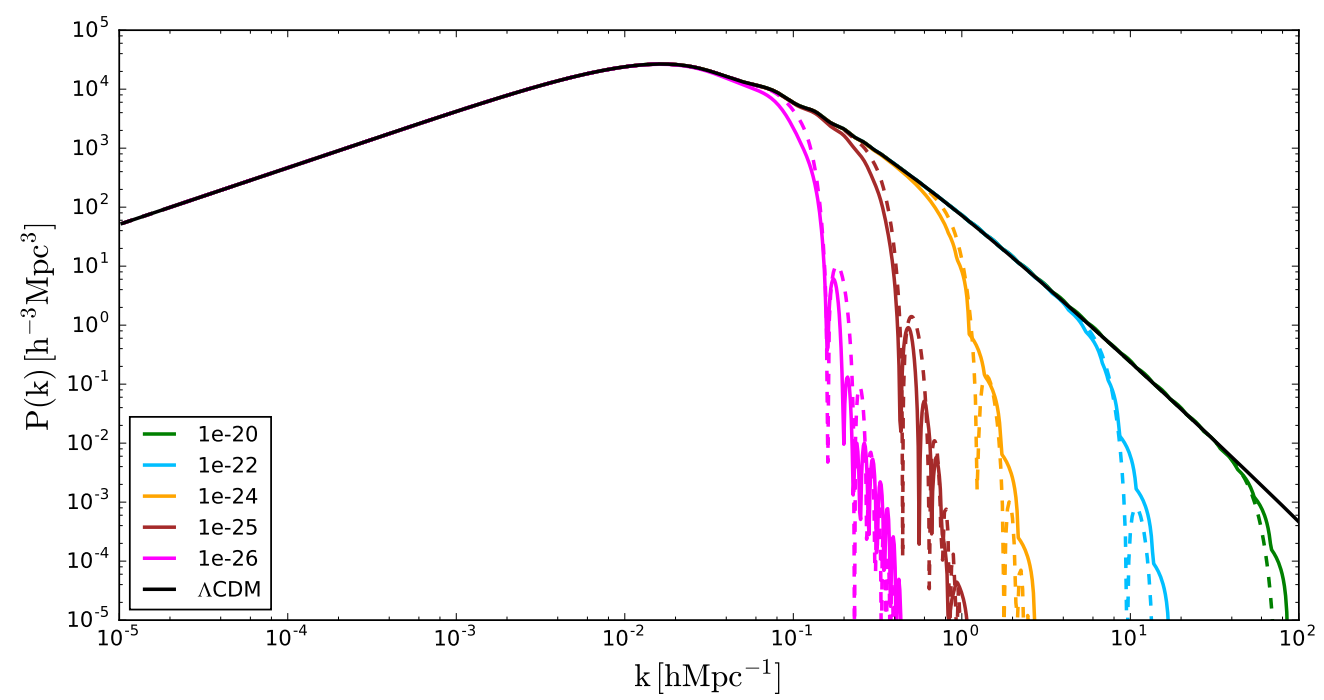

Figure 3. Mass PS for different masses of the scalar field, and for the $\Lambda$ CDM model (black line), as obtained from the amended version of CLASS. The most noticeable effect is the well-known cutoff at large scales (small k's) for the lightest mass considered. Also shown for comparison is the approximated power spectrum using equation (3.13) (dashed lines), for the corresponding scalar field mass. Qualitatively the two approaches are consistent, see the text for a more quantitative discussion.

for the lightest masses considered for which differences are more than noticeable. As for the matter spectrum, the most remarkable feature is the expected sharp cut-off for the smallest scales, which is clearly seen in the cases $m \lesssim 10^{-24} \mathrm{eV}$.

One known approach to calculate the mass power spectrum for the SFDM model was to rescale the $\Lambda \mathrm{CDM}$ one using the expression:

$$
P_{\mathrm{SFDM}}(k, z)=T^{2}(k) P_{\Lambda \mathrm{CDM}}(k, z)
$$

where the transfer function $T(k)$ is effectively considered as redshift independent:

$$
T(k)=\frac{\cos \left(x^{3}\right)}{\left(1+x^{8}\right)}, \quad x=1.61\left(\frac{m}{10^{-22} \mathrm{eV}}\right)^{1 / 18} \frac{k}{k_{J, e q}},
$$

and the Jeans scale reads $k_{J, e q}=9 m_{22}^{1 / 2} \mathrm{Mpc}^{-1}$. In Fig. 3, we show the SFDM PS calculated from Eq. (3.13) and the one calculated numerically in this work, and both of them are compared with the $\Lambda \mathrm{CDM}$ one. We can notice that the cut-off seem to match well in both approaches, and our result is in agreement with those already reported in the literature $[14,18,20,22,23,39,75,76]$. We shall discuss the differences more in detail in Sec. 5 below.

\section{Suppression of power in scalar field perturbations}

The common explanation for the suppression of power in the mass spectrum of SFDM is usually given in terms of the Jeans wave number (3.6), but still this is a confusing subject in the literature, mostly because of the different by-hand manipulations in the fluid approximation of the equations of motion. To illustrate the point, notice that we can write: 
$k^{2} / k_{J}^{2}=\left(k^{2} / a^{2} m^{2}\right)(m / H)$, and then previous works have made separate and complicated studies of the perturbations in terms of the ratios $k / a m$ and $m / H[18,22,39,41]$. As we are to explain now, the linear equations (3.11) provide a simpler, though a bit subtler, explanation for the suppression of linear perturbations. The semi-analytic results below will be compared with the numerical ones obtained from the amended version of CLASS, which are shown in Figs. 4, 5 and 6 below.

\subsection{General form of the equations of motion for scalar field perturbations}

For a better visualization of the physics behind the evolution of scalar field perturbations, it is convenient to write the equations of motion (3.11) in the following form:

$\left.\left[\begin{array}{c}e^{\alpha} \cos (\tilde{\vartheta} / 2) \\ e^{\alpha} \sin (\tilde{\vartheta} / 2)\end{array}\right]^{\prime}=-\left[\begin{array}{cc}3 \cos \theta+\tilde{\omega} \sin \theta & \tilde{\omega}(1+\cos \theta) \\ 3 \sin \theta+\tilde{\omega}(1-\cos \theta) & -\tilde{\omega} \sin \theta\end{array}\right]\left[\begin{array}{c}e^{\alpha} \cos (\tilde{\vartheta} / 2) \\ e^{\alpha} \sin (\tilde{\vartheta} / 2)\end{array}\right]+\frac{h^{\prime}}{2}\left[\begin{array}{c}\sin \theta \\ 1-\cos \theta\end{array}\right]+.1\right)$

where $\tilde{\omega}=\omega / 2$. To find the solutions of the field perturbations at early times, before the onset of the field oscillations, we just set $\theta=0$ in the $2 \times 2$ matrix, and retain the lowest order terms of $\theta$ in the last vector on the rhs of Eq. (4.1). The latter then becomes:

$$
\left[\begin{array}{c}
e^{\alpha} \cos (\tilde{\vartheta} / 2) \\
e^{\alpha} \sin (\tilde{\vartheta} / 2)
\end{array}\right]^{\prime} \simeq-\left[\begin{array}{ll}
3 & \tilde{\omega} \\
0 & 0
\end{array}\right]\left[\begin{array}{c}
e^{\alpha} \cos (\tilde{\vartheta} / 2) \\
e^{\alpha} \sin (\tilde{\vartheta} / 2)
\end{array}\right]+\frac{h^{\prime}}{2}\left[\begin{array}{c}
\theta \\
\theta^{2} / 2
\end{array}\right] .
$$

If we again consider that during RD: $h=h_{i}\left(a / a_{i}\right)^{2}$ and $\theta=\theta_{i}\left(a / a_{i}\right)^{2}$, the growing solutions of Eq. (4.2) are:

$$
\left[\begin{array}{c}
e^{\alpha} \cos (\tilde{\vartheta} / 2) \\
e^{\alpha} \sin (\tilde{\vartheta} / 2)
\end{array}\right] \simeq h \theta\left[\begin{array}{c}
1 / 7 \\
\theta / 12
\end{array}\right]
$$

In agreement with the results in Sec. 3.1, inhomogeneities in the scalar field are then negligible at early times, mostly because $\theta$ diminishes the influence of the driving term in Eq. (4.1). This situation can only be changed once the field approaches the onset of oscillations at $\theta=\pi / 2$. Hence, we see that one reason for the suppression of power in the matter spectrum is the late appearance of the oscillations of the scalar field, and clearly such suppression is more acute for smaller values of the field mass $m$. This is a general effect that happens for all scales, as Eq. (4.2) is independent on the wavenumber $k$.

\subsection{General solution of perturbations after the onset of the field oscillations}

Once the field oscillations start, any trigonometric function that depends on $\theta$ must be considered a rapidly oscillating function. Following our cut-off procedure ${ }^{9}$ (see Eq. (2.18)), we shall consider that: $\sin _{\star} \theta \rightarrow 0$ and $\cos _{\star} \theta \rightarrow 0$. Without such rapidly-oscillating trigonometric functions, Eq. (4.1) now becomes:

$$
\left[\begin{array}{c}
e^{\alpha} \cos (\tilde{\vartheta} / 2) \\
e^{\alpha} \sin (\tilde{\vartheta} / 2)
\end{array}\right]^{\prime} \simeq \tilde{\omega}\left[\begin{array}{cc}
0 & 1 \\
-1 & 0
\end{array}\right]\left[\begin{array}{c}
e^{\alpha} \cos (\tilde{\vartheta} / 2) \\
e^{\alpha} \sin (\tilde{\vartheta} / 2)
\end{array}\right]+\frac{h^{\prime}}{2}\left[\begin{array}{l}
0 \\
1
\end{array}\right] .
$$

Eq. (4.4a) has the form of a forced harmonic oscillator with characteristic frequency $\tilde{\omega}$. In order to find approximate solutions, we will assume that $\tilde{\omega}=$ const. (which is only strictly true during radiation domination), and then Eq. (4.4a) formally integrates into:

$$
\left[\begin{array}{c}
e^{\alpha} \cos (\tilde{\vartheta} / 2) \\
e^{\alpha} \sin (\tilde{\vartheta} / 2)
\end{array}\right] \simeq\left[\begin{array}{cc}
\cos (\tilde{\omega} N) & \sin (\tilde{\omega} N) \\
-\sin (\tilde{\omega} N) & \cos (\tilde{\omega} N)
\end{array}\right]\left[\begin{array}{l}
C_{1} \\
C_{2}
\end{array}\right]+\frac{1}{2} \int_{0}^{N} d \hat{N} h^{\prime}\left[\begin{array}{c}
\sin [\tilde{\omega}(N-\hat{N})] \\
\cos [\tilde{\omega}(N-\hat{N})]
\end{array}\right]
$$

\footnotetext{
${ }^{9}$ The influence of the rapidly oscillating terms in the solution of Eq. (4.1) is explained in the Appendix A.3, where it is shown that the solution at late times coincides with that of Eq. (4.4b).
} 
where $C_{1}, C_{2}$ are integration constants, and $N=\ln \left(a / a_{o s c}\right)$. The integral on the rhs of Eq. (4.4b) can be made only if we know the form of the metric continuity $h^{\prime}$, which in turn can only be found after solving the full set of perturbation equations for all the matter fields.

As we are going to show now, a shortcut is to assume that we somehow know the behavior of the metric term $h$. In general, we will have two possibilities. The first one is to consider that $h=h_{0} e^{\beta N}$, where $\beta>0$ is a constant, and $h_{0}$ is the initial value of $h$. For instance, in the standard $\Lambda \mathrm{CDM}$ model we find that the growing solutions of the metric perturbations are of the form $h=h_{0} e^{4 N}\left(h=h_{0} e^{N}\right)$ during RD (MD), and then we can assume that $h^{\prime}=\beta h_{0} e^{\beta N}$. Likewise, in the case that matter perturbations are suppressed ${ }^{10}$ we then expect that $h \simeq h_{\infty}+\left(h_{0}-h_{\infty}\right) e^{\beta N}$, here $h_{0}\left(h_{\infty}\right)$ are the values at $N \rightarrow 0(N \rightarrow \infty)$; in this case $\beta<0$ and then $h^{\prime}=\beta\left(h_{0}-h_{\infty}\right) e^{\beta N}$. The general solution of Eq. (4.4b), in either of the aforementioned cases, can be written as:

$$
\left[\begin{array}{c}
e^{\alpha} \cos (\tilde{\vartheta} / 2) \\
e^{\alpha} \sin (\tilde{\vartheta} / 2)
\end{array}\right] \simeq\left[\begin{array}{cc}
\cos (\tilde{\omega} N) & \sin (\tilde{\omega} N) \\
-\sin (\tilde{\omega} N) & \cos (\tilde{\omega} N)
\end{array}\right]\left[\begin{array}{c}
C_{1}-\frac{\tilde{\omega}}{2\left(\beta^{2}+\tilde{\omega}^{2}\right)} h_{0}^{\prime} \\
C_{2}-\frac{\beta}{2\left(\beta^{2}+\tilde{\omega}^{2}\right)} h_{0}^{\prime}
\end{array}\right]+\frac{h^{\prime}}{2\left(\beta^{2}+\tilde{\omega}^{2}\right)}\left[\begin{array}{c}
\tilde{\omega} \\
\beta
\end{array}\right] .
$$
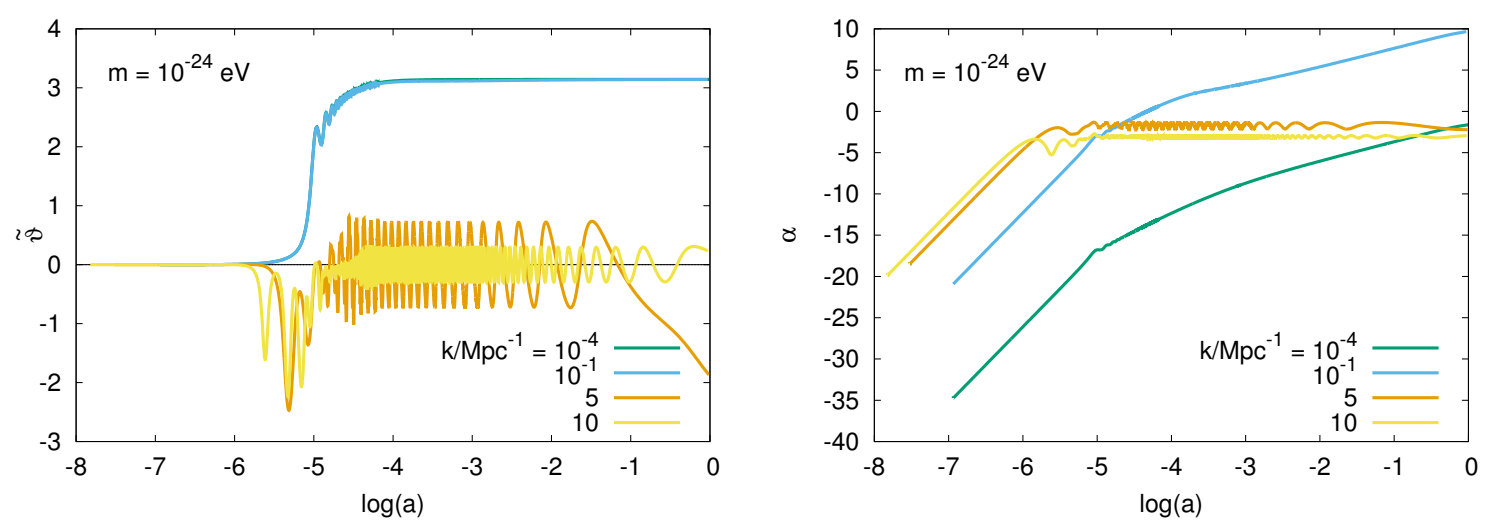

Figure 4. Numerical solutions for the perturbation variables $\tilde{\vartheta}$ (left) and $\alpha$ (right) for an example with a mass $m=10^{-24} \mathrm{eV}$. For each variable we show two growing and two decaying cases, following the definitions in Sec. 4.2, which correspond to the wavenumbers $k=10^{-4}, 10^{-1} \mathrm{Mpc}^{-1}$, and $k=5,10 \mathrm{Mpc}^{-1}$, respectively. The Jeans wavenumber for the given mass is $k_{J 0}=1.8 \mathrm{Mpc}^{-1}$, see Eq. (3.6b), and then the cases can also be distinguished by the value of the frequency $\omega$ : for the growing case $\omega \ll 1$, whereas $\omega \gg 1$ for the decaying one. In general, we can observe that for the growing case $\tilde{\vartheta} \rightarrow \pi$ (note that both green and blue lines coincide on the left panel) and $\alpha$ grows, whereas for the decaying case both $\tilde{\vartheta}$ and $\alpha$ become oscillatory functions. These behaviors are in very good agreement with those inferred from the semi-analytical results in Eqs. (4.4) and (4.5). In particular, we can see by eye that the solutions for small scales oscillate in terms of the number of $e$-folds $N$. However, the numerical solutions show that the frequency of the oscillations decreases as the evolution proceeds, which is expected as $\tilde{\omega}$ decreases after the epoch of RD. See the text for more details.

The general solution then consists of an oscillating part plus an extra term proportional to the driving term containing the metric perturbation. Before studying the general cases, we analyze the forced solutions without the oscillating terms. The separate solutions of the

\footnotetext{
${ }^{10}$ Such behavior of the metric perturbation $h$ is expected if the perturbed Einstein equations are not sourced at all by any density perturbations, see for instance Eqs. (21) in[77].
} 
variables $\tilde{\vartheta}$ and $e^{\alpha}$ are, in full generality, given by:

$$
\tan (\tilde{\vartheta} / 2) \simeq \frac{\beta}{\tilde{\omega}}, \quad e^{\alpha} \simeq \frac{h^{\prime}}{2\left(\beta^{2}+\tilde{\omega}^{2}\right)^{1 / 2}} .
$$

As we shall see now, there are only two consistent solutions if the scalar field perturbations are going to be the main source of matter perturbations.

The first one is what we call the growing case, which is achieved if $\omega \ll 1$ and $e^{\alpha}$ is a growing function. From the discussion above, this case corresponds to $h^{\prime}=\beta h$, and then:

$$
\tilde{\vartheta} \simeq \pi-2 \tilde{\omega} / \beta, \quad e^{\alpha} \simeq \frac{1}{2} h\left(1-\tilde{\omega}^{2} / \beta^{2}\right),
$$

where we have included the correction at the lowest order in $\tilde{\omega} / \beta \ll 1$. The first thing to notice is that the angular variable $\tilde{\vartheta} \rightarrow \pi$, and also $e^{\alpha} \rightarrow h / 2$. In consequence, the scalar field density contrast at late times, from Eq. (3.4a), can be written as $\delta_{\phi} \simeq-(h / 2) \cos (\tilde{\omega} / \beta) \simeq$ $-h / 2$ (notice that this resembles the functional form of the CDM growing mode solution, which is $\delta_{\mathrm{CDM}}=-h / 2$ exactly). The solution is consistent in the sense that both the field perturbations and the metric continuity are growing functions. The numerical solutions corresponding to this case are shown in Fig. 4, for the wavenumbers $k=10^{-4}, 10^{-1} \mathrm{Mpc}^{-1}$ and a scalar field mass $m=10^{-24} \mathrm{eV}$.

The second possibility is the decaying case, for which $\tilde{\omega} \gg 1$ and $e^{\alpha}$ is a non-growing function. The latter condition can be achieved in the second case discussed above corresponding to $h^{\prime}=\beta\left(h_{0}-h_{\infty}\right) e^{\beta N}$, from which we obtain that:

$$
\tilde{\vartheta} \simeq 2 \beta / \tilde{\omega}, \quad e^{\alpha} \simeq \frac{\beta / \tilde{\omega}}{2\left(1+\beta^{2} / \tilde{\omega}^{2}\right)^{1 / 2}}\left(h_{\infty}-h\right),
$$

where we included again the correction at the lowest order in $\beta / \tilde{\omega} \ll 1$. Eq. (4.5c) suggests that this time the angular variable $\tilde{\vartheta} \simeq 0$ for $|\beta / \tilde{\omega}| \ll 1$, and that the amplitude of perturbations vanishes, $e^{\alpha} \rightarrow 0$, as $h \rightarrow h_{\infty}$. The two results together imply that $\delta_{\phi} \rightarrow 0$. Like in the growing case, the new solution is also consistent in the sense that both the metric and field perturbations are non-growing functions.

However, as the driving term in Eq. (4.4c) vanishes at late times for the decaying case, the true solution is eventually represented by its oscillatory part only. This is shown by the numerical solutions in Fig. 4 for the wavenumbers $k=5,10 \mathrm{Mpc}^{-1}$, as it is for these values of $k$ that $\omega \gg 1$ if $m=10^{-24} \mathrm{eV}$. As a final note, we must mention that the case with $k=5 \mathrm{Mpc}^{-1}$ shows that $\tilde{\vartheta}$ slowly drifts towards $-\pi$ at late times ${ }^{11}$. This is to be expected, given that our previous assumption that $\omega=$ const. is not strictly attained during the whole evolution. Actually, we can see that the frequency of the oscillations (with respect to the variable $N$ ) diminishes at late times, just because the Jeans wavenumber $k_{J}$ is a growing function after the time of radiation-matter equality (see the discussion around Eq. (3.6a)). This is an interesting example in which one given scale starts in the regime $\tilde{\omega} \gg 1$, but changes to the one with $\tilde{\omega} \ll 1$ at late times.

Apart from the dynamical variables $\alpha, \tilde{\vartheta}$, there are two quantities that are of more physical interest: the scalar field density contrast $\delta_{\phi}$, and the velocity gradient $\Theta_{\phi}$. According to Eq. (3.4a), the density contrast can be written as $\delta_{\phi}=-e^{\alpha} \sin (\tilde{\vartheta} / 2)$, and then its general

\footnotetext{
${ }^{11}$ Notice that $\tilde{\vartheta} \rightarrow-\pi$, instead of $\tilde{\vartheta} \rightarrow \pi$, in the case $k=5 \mathrm{Mpc}^{-1}$. This is not but a numerical glitch because CLASS cannot in this case keep an accurate calculation of the oscillations of $\tilde{\vartheta}$ at late times.
} 
evolution can be obtained directly from the solutions presented above. To determine the general behavior of the velocity gradient $\Theta_{\phi}$, let us write Eq. (3.4c) in the form

$$
\left(\rho_{\phi}+p_{\phi}\right) \Theta_{\phi}=\rho_{\phi} \Theta_{\phi}(1-\cos \theta)=-\frac{k^{2}}{a m} \rho_{\phi} e^{\alpha} \sin (\theta / 2) \sin (\vartheta / 2),
$$

where we have used the EoS from Eq. (2.6). We can see that the source of SFDM velocity perturbations is indeed a rapidly oscillating function at late times. As we shall see, though, the important point is to determine whether those oscillations are relevant or not by calculating their amplitude, which is represented by the product $\rho_{\phi} \Theta_{\phi}$. At early times we can write Eq. (4.6) as:

$$
\frac{1}{2} \rho_{\phi} \Theta_{\phi} \theta^{2} \simeq-\frac{5 k^{2}}{24 a m} \rho_{\phi} e^{\alpha} \theta^{2} \rightarrow \rho_{\phi} \Theta_{\phi} \simeq-\frac{5 k^{2}}{12 a m} \rho_{\phi} e^{\alpha},
$$

whereas at late times, after the onset of the field oscillations, we can use that $\vartheta \simeq \theta=2 m t$ and then Eq. (4.6) can be written as:

$$
2 \rho_{\phi} \sin ^{2}(m t) \Theta_{\phi} \simeq-\frac{k^{2}}{a m} \rho_{\phi} e^{\alpha} \sin ^{2}(m t) \quad \rightarrow \quad \rho_{\phi} \Theta_{\phi} \simeq-\frac{k^{2}}{2 a m} \rho_{\phi} e^{\alpha} .
$$

Hence, we will take the last term in Eq. (4.7b) as a good approximation at all times for the product $\rho_{\phi} \Theta_{\phi}$. In the following sections, we will analyze the behavior of the perturbation quantities $\delta_{\phi}$ and $\rho_{\phi} \Theta_{\phi}$ for large and small scales as compared to the Jeans wavenumber $k_{J}$.

\subsection{Large scales $k \ll k_{J}$}

For large enough scales $k \ll k_{J}$, the characteristic frequency of the oscillator (4.4a) is small at all times: $\omega \ll 1$. According to our discussion above, the only consistent solution for this case is the growing one represented by Eq. (4.5b), and then the complete solution for the scalar field density contrast, from Eq. (4.4c), is:

$$
\delta_{\phi} \simeq-\left[C_{1}-\frac{\beta^{2}}{2\left(\beta^{2}+\tilde{\omega}^{2}\right)} h_{0}\right] \sin (\tilde{\omega} N)-\left[C_{2}-\frac{\beta \tilde{\omega}}{2\left(\beta^{2}+\tilde{\omega}^{2}\right)} h_{0}\right] \cos (\tilde{\omega} N)-\frac{\beta^{2}}{2\left(\beta^{2}+\tilde{\omega}^{2}\right)} h .
$$

If in addition $\omega \ll \beta$, this solution indicates that at late times, $\delta_{\phi} \simeq-h / 2$, with some superimposed oscillations of small amplitude. Thus, SFDM behaves effectively as a pressureless component at the level of linear perturbations for large scales. This can also be corroborated from the calculation of the pressure contrast in Eq. (3.4b): $\delta_{p_{\phi}}=-e^{\alpha} \sin (\theta+\tilde{\vartheta} / 2)$, from which we see that it is a rapidly oscillating function that becomes zero after the cut-off of the oscillations.

On the other hand, we see that the asymptotic solution of the amplitude of SFDM velocity perturbations at late times is:

$$
\rho_{\phi} \Theta_{\phi} \simeq-\frac{k^{2}}{4 a m} \frac{\tilde{\omega}}{\beta} h \rho_{\phi} \simeq-\frac{k^{2} h_{\text {osc }}}{4 m a_{\text {osc }}} \frac{\tilde{\omega}}{\beta} \rho_{\phi} \simeq \text { const. } \times \tilde{\omega} a^{-3},
$$

where we have used Eq. (4.5b), and the last term appears if $h=h_{o s c} e^{N}=h_{o s c}\left(a / a_{o s c}\right)$, which is the expected expression during matter domination. Eq. (4.8b) then indicates that there must be a residual amplitude of the velocity gradient at late times, which is quite small because of the assumption here that $\tilde{\omega} \ll \beta\left(k \ll k_{J}\right)$. Moreover, the residual amplitude 

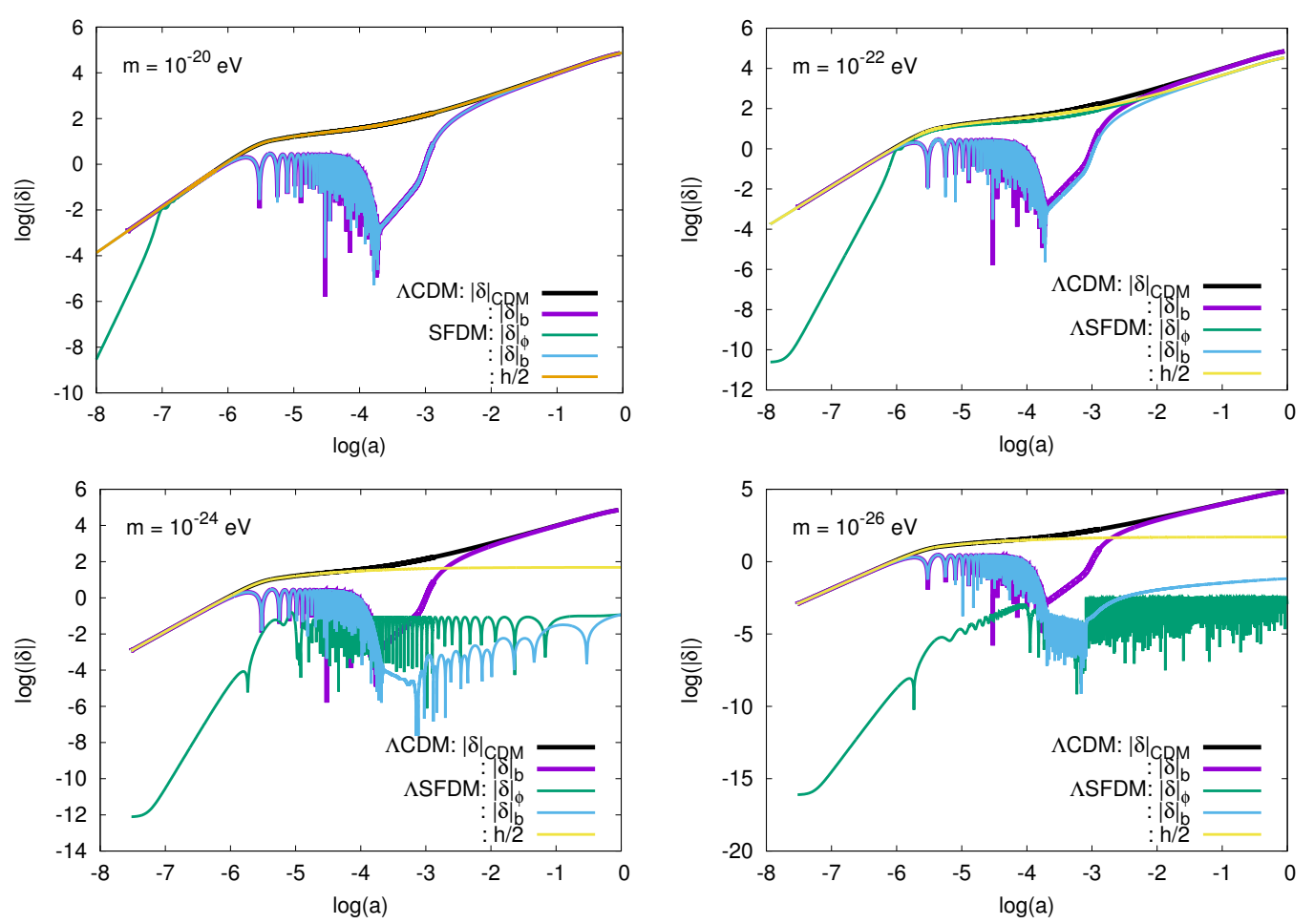

Figure 5. The evolution of the density contrast of CDM, baryons (b), and SFDM $(\phi)$ for the same wavenumber $k=5 \mathrm{Mpc}^{-1}$, as obtained from the amended version of CLASS for the different scalar field masses indicated in each one of the plots. As explained in the Secs. 4.3 and 4.4 below, the important parameter for the suppression of the scalar field perturbations is the ratio $\tilde{\omega}=k^{2} / 2 k_{J}^{2}$, and then we refer to large (small) scales to the cases in which $\tilde{\omega} \ll 1(\tilde{\omega} \gg 1)$. Given that this ratio depends on the mass of the scalar field, one same scale can give $\tilde{\omega} \ll 1$ for large masses (e.g. $m=10^{-20}, 10^{-22} \mathrm{eV}$ ), or $\tilde{\omega} \gg 1$ for small masses (e.g. $m=10^{-24}, 10^{-26} \mathrm{eV}$ ). The latter case also means that there are some residual oscillations with frequency $\tilde{\omega}$ apart from the high-frequency ones related to $\theta$ that were cut-off in the modified version of CLASS, see Eq. (4.10) and Fig. 4

. In general, SFDM perturbations evolve as those of CDM for the large scales (see Sec. 4.3), whereas they appear suppressed for the small scales (see Sec. 4.4). Also shown is the evolution of the metric perturbation $h / 2$ (see Eq. (3.1)), which for late times accomplishes the relation $\delta_{\phi}=-h / 2$ for large scales, whereas for small scales it approaches an asymptotic value: $h \rightarrow h_{\infty}$. This is to be compared with the usual result $\delta_{\mathrm{CDM}}=-h / 2$, which is exact for all scales in the case of CDM[77]. See the text for more details.

decays as $\tilde{\omega} a^{-3}$, where we must take into account that $\tilde{\omega}$ also contributes with an extra factor of $a^{-1} H^{-1 / 2} \sim a^{-1 / 4}$ during matter domination.

It must be recalled that the condition for large scales $k \ll k_{J}$ depends upon the value of the scalar field mass. In Figs. 5 and 6 we give numerical examples obtained from CLASS of the behavior of the density contrast $\delta_{\phi}$ and of the velocity perturbation source term $\left(\rho_{\phi}+p_{\phi}\right) \Theta_{\phi}$, respectively, in comparison with those of other matter components. The large scale case corresponds, in both figures, to the masses $m=10^{-20}, 10^{-22} \mathrm{eV}$, and it can be clearly seen that both physical quantities evolve as suggested by the approximate solutions (4.8).

We have said before that the semi-analytical results suggest that for large scales $\delta_{\phi} \simeq$ $-h / 2$, just as for any cold matter component. This does not mean, however, that the 

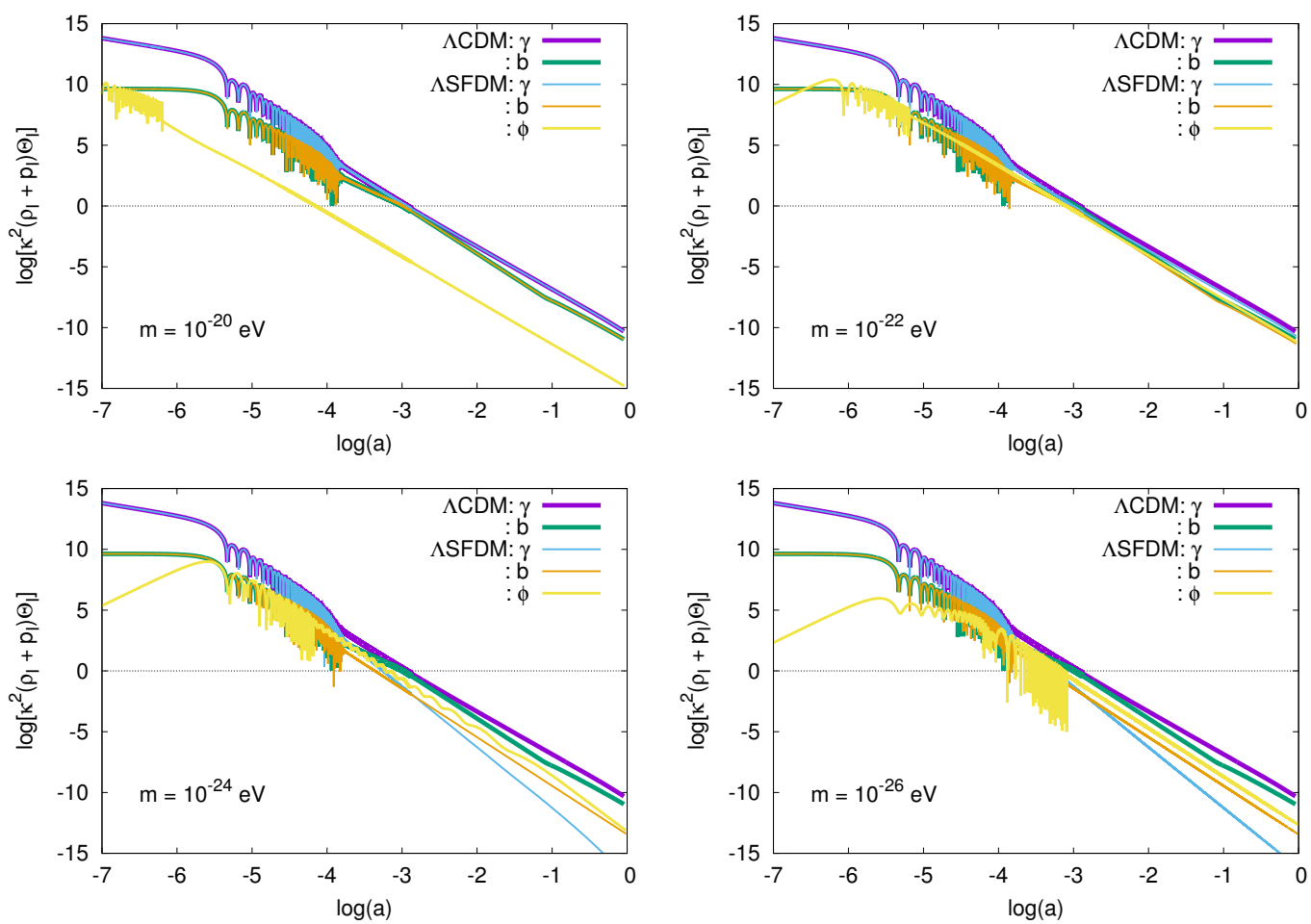

Figure 6. The amplitude of the velocity perturbation source term $\left(\rho_{I}+p_{I}\right) \Theta_{I}$ of different matter components for the same wavenumber $k=5 \mathrm{Mpc}^{-1}$ as in Fig. 5. That of a CDM component is not shown, as it is zero by definition in the synchronous gauge. The velocity gradient of photons and baryons, in the presence of SFDM, is the same as in $\Lambda \mathrm{CDM}$ for large scales (top figures, for $m=10^{-20}, 10^{-22} \mathrm{eV}$ ), but they seem to decay more rapidly for small scales (bottom figures, for $m=10^{-24}, 10^{-26} \mathrm{eV}$ ) at late times. It can be confirmed that SFDM velocity perturbations evolve as expected of a pressureless component in the frame comoving with a CDM component, which is indeed the gauge considered within CLASS for the calculation of perturbations. As a side effect, notice that velocity gradient of the photons is further suppressed at late times, as compared to that of the $\Lambda \mathrm{CDM}$ model, which is an expected result if $h^{\prime} \rightarrow 0$ (see Eqs. (29) in[77]). For more details, see the text in Secs. 3.2, 4.3, and 4.4.

amplitude of the SFDM density contrast is the same as in the CDM case. This can be noticed in Fig. 5 for $m=10^{-22} \mathrm{eV}$, in which case the given wavenumber is mildly larger than the Jeans length: $k>k_{J}$, and in consequence $\delta_{\phi}$ has a bit smaller amplitude than $\delta_{C D M}$.

As for the velocity gradient shown in Fig. 6 , the same case with $m=10^{-22} \mathrm{eV}$ shows a brief stage of rapid oscillation, which is also seen to disappear because of the cut-off imposed upon the rapidly oscillating trigonometric functions in our amended version of CLASS.

\subsection{Small scales $k \gg k_{J}$}

For the case of small enough scales, $k \gg k_{J}$, corresponding to $\omega \gg 1$, the consistent solution at late times for the density contrast, from Eq. (4.4c), is:

$$
\delta_{\phi} \simeq-\left[C_{1}-\frac{\tilde{\omega} \beta\left(h_{0}-h_{\infty}\right)}{2\left(\beta^{2}+\tilde{\omega}^{2}\right)}\right] \sin (\tilde{\omega} N)+\left[C_{2}-\frac{\beta^{2}\left(h_{0}-h_{\infty}\right)}{2\left(\beta^{2}+\tilde{\omega}^{2}\right)}\right] \cos (\tilde{\omega} N)+\frac{\beta^{2}\left(h_{\infty}-h\right)}{2\left(\beta^{2}+\tilde{\omega}^{2}\right)},
$$

In contrast to the case of large scales, this time the driving term decreases as $h \rightarrow h_{\infty}$ and does not become the dominant part of the solution. Instead, in the limit $\beta / \tilde{\omega} \ll 1$, we find 
that:

$$
\delta_{\phi} \simeq-C_{1} \sin (\tilde{\omega} N)+C_{2} \cos (\tilde{\omega} N) .
$$

Hence, the density contrast turns into a (rapidly) oscillating function of $N$ with a small amplitude, with the latter inherited from the transition to the rapidly oscillating phase of the scalar field. As for the SFDM velocity perturbations, see Eq. (4.6), because $e^{\alpha} \rightarrow$ const. their amplitude quickly decreases as $\rho_{\phi} \Theta_{\phi} \simeq\left(k^{2} / 2 a m\right) \rho_{\phi} e^{\alpha} \sim a^{-4}$. Together with the results in the previous section, this reinforces our argument in Sec. 3.2 that the velocity gradient of SFDM evolves as expected of a pressureless component in the gauge corresponding to the comoving frame of a CDM component.

Numerical examples for small scales are also shown in Figs. 5 and 6 for the masses $m=$ $10^{-24}, 10^{-26} \mathrm{eV}$, and they agree very well with the behavior inferred from the approximate solutions described just above: the density contrast is just an oscillating function with a tiny amplitude, whereas the velocity gradient oscillates with a rapidly decaying amplitude.

\section{Power spectrum and mass function}

In order to exploit the constraining power of future galaxy surveys such as DESI[80] and LSST[81], it will be necessary to perform an accurate modeling of the linear, and non linear, matter PS of any given dark matter model, for wavenumbers up to $k \sim 10 \mathrm{Mpc}^{-1}$. For $\Lambda$ CDM models, linear perturbation theory provides good accuracy up to scales of $k \sim$ $0.1 \mathrm{~h} \mathrm{Mpc}^{-1}$, whereas dark matter-only N-body simulations provide good results up to $k \sim$ $0.5 \mathrm{~h} \mathrm{Mpc}^{-1}$, for larger values of $k$ it is necessary to include baryonic physics in the simulations [82].

For the SFDM model, we are able to describe the linear PS at scales that can be considered in the semi-linear regime without any by-hand approximation. A correct treatment of the linear and semi-linear regime impacts the study of the structure formation process in two aspects: on setting up the initial conditions for cosmological simulations, and on the prediction (as a first approach) of observables about large and small scale structure.

Initial conditions for structure formation simulations are set up at high redshift using the predicted PS for a given cosmology. In the case of SFDM, two approaches have been so far considered for the task. The first one starts a cosmological simulation at a very high redshift $z \approx 1000$, when DM is just to dominate the expansion of the Universe. For this case, we compare the PS used to set up the initial conditions in[51], to the one calculated from our amended CLASS code. The resultant PS are shown in Fig. 7, for two different values of the SF mass at different redshifts: $z=0,47,200,1000$. A comparison with figure S2 in[51], we note that for the two versions of the PS the amplitude of the first oscillation matches quite well, but for smaller scales the amplitudes seem to be overestimated in the simulation, or likewise underestimated by us. At this point, though, it is not possible to tell which of the approaches is the correct one, since the CMB codes used are different: CMBFAST was used in[51], which is a code not longer supported by their developers. However, the differences, mostly present at the largest values of $k$, might be important only if the resolution in the simulation is of the order of the Jeans scale.

The second approach is to use Eq. 3.13 to just re-scale the $\Lambda$ CDM PS. In Fig. 8, we show the PS obtained from our modified CLASS code (solid lines) and the re-scaled $\Lambda$ CDM one (dashed lines), for two SFDM masses and for different redshifts. Relative differences between the two PS are redshift independent for the scales shown, but for the first oscillation they can be as large as $40 \%$ if $m=10^{-22} \mathrm{eV}$, and almost as $100 \%$ if $m=10^{-23} \mathrm{eV}$. For the 

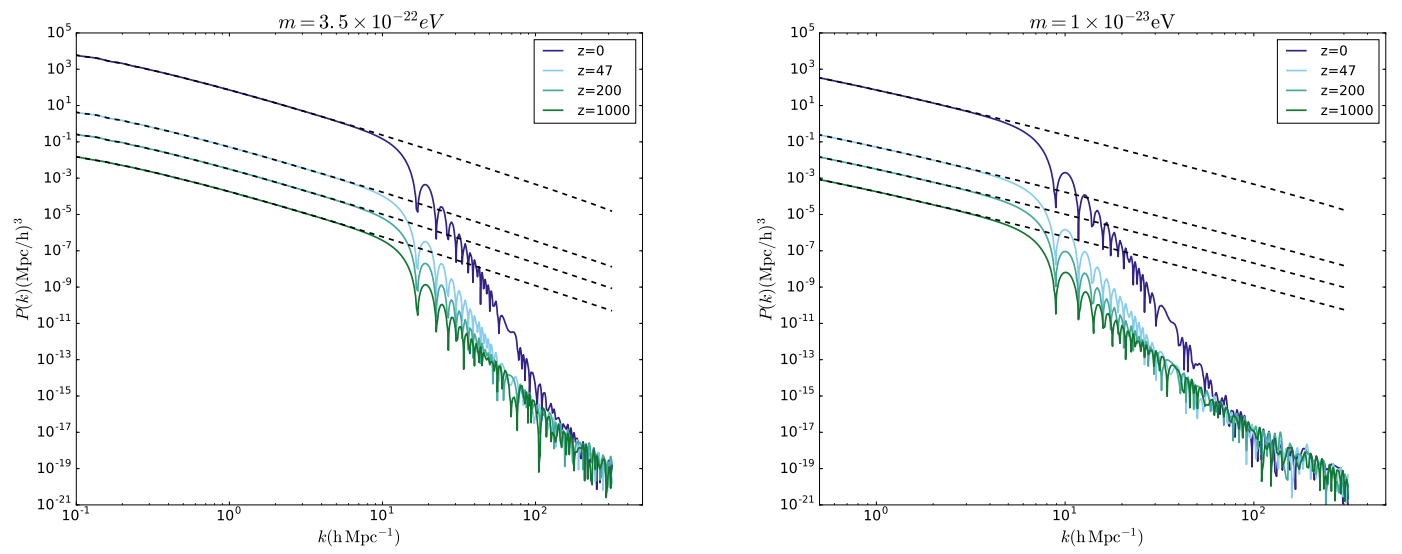

Figure 7. Mass PS for two values of the scalar field mass that resemble the initial condition of the cosmological simulation presented in Ref. [51] (see figure S2 of that paper). Although we cannot make a precise comparison, the amplitude of the first oscillations seem to be in good agreement, but for lager values of $k$ the amplitude of the PS differ noticeably even by 9 orders of magnitude, which means that perturbations are much more suppressed than the estimation made in[51]. This difference might be important only if there is enough resolution in the simulations to include such $k$-values

values of $k$ in the oscillatory regime, the differences can be at least of $20 \%$. It seems then that using the approximated PS of3.13 could be introducing a significant error, even though it predicts correctly the scale at which the PS starts to oscillate.

On the other hand, for observables lying at high, medium, or low redshifts, one could use the approximate form of the PS given by Eq. 3.13, as used in [51], or the numerical output from our amended version of CLASS. In Fig. 9 we compare our resultant PS to the LCDM one, and take the difference as an estimate of the precision needed in an observation in order to constrain the model. SFDM models with masses larger than $10^{-22} \mathrm{eV}$ seems not to be discriminated from the $\Lambda \mathrm{CDM}$ model, by the PS shape obtained from current and next generation of galaxy surveys (BOSS/PS and DESI/PS). However, the region in between $10^{-23}-10^{-22} \mathrm{eV}$ could be proved by the Lyma- $\alpha$ forest data (BOSS/Ly- $\alpha$ and DESI/Ly- $\alpha$ ) only if information for larger $k$ is included, such as the data from HIRES and MIKE, as was used in [83]. Notice that for Warm Dark Matter (WDM) models there is a strong constraint on the suppression of the linear matter power spectrum at (non-linear) scales corresponding to $k=10 \mathrm{~h} \mathrm{Mpc}^{-1}$, which should not deviate more than $10 \%$ from that of $\Lambda \mathrm{CDM}$ model. For the SFDM this implies a strong constraint up to masses of $\sim 10^{-21} \mathrm{eV}$. However one should take this constraint with care because it lays at highly no-linear scales, and it could strongly depend on the non-linear evolution of the SFDM perturbations in the presence of baryons.

Notice, also from 9, that the LSST survey could, at the very end of its $k$ coverage, set some constraints directly from the PS at the semi-linear regime. This is a very simple estimation based on [85] where they forecast the power of constraining the primordial PS. If we relay on standard assumptions about the primordial PS then constraints could be set SFDM PS. A detailed forecast analysis will be done elsewhere, however it is clear it will not be easy to discriminate between the effect of a modification to the primordial PS or modifications due to SFDM. The information from other observables will be necessary. LSST will probably set more constraints through weak and strong lensing, at smaller scales (larger $k$ 's), but such 

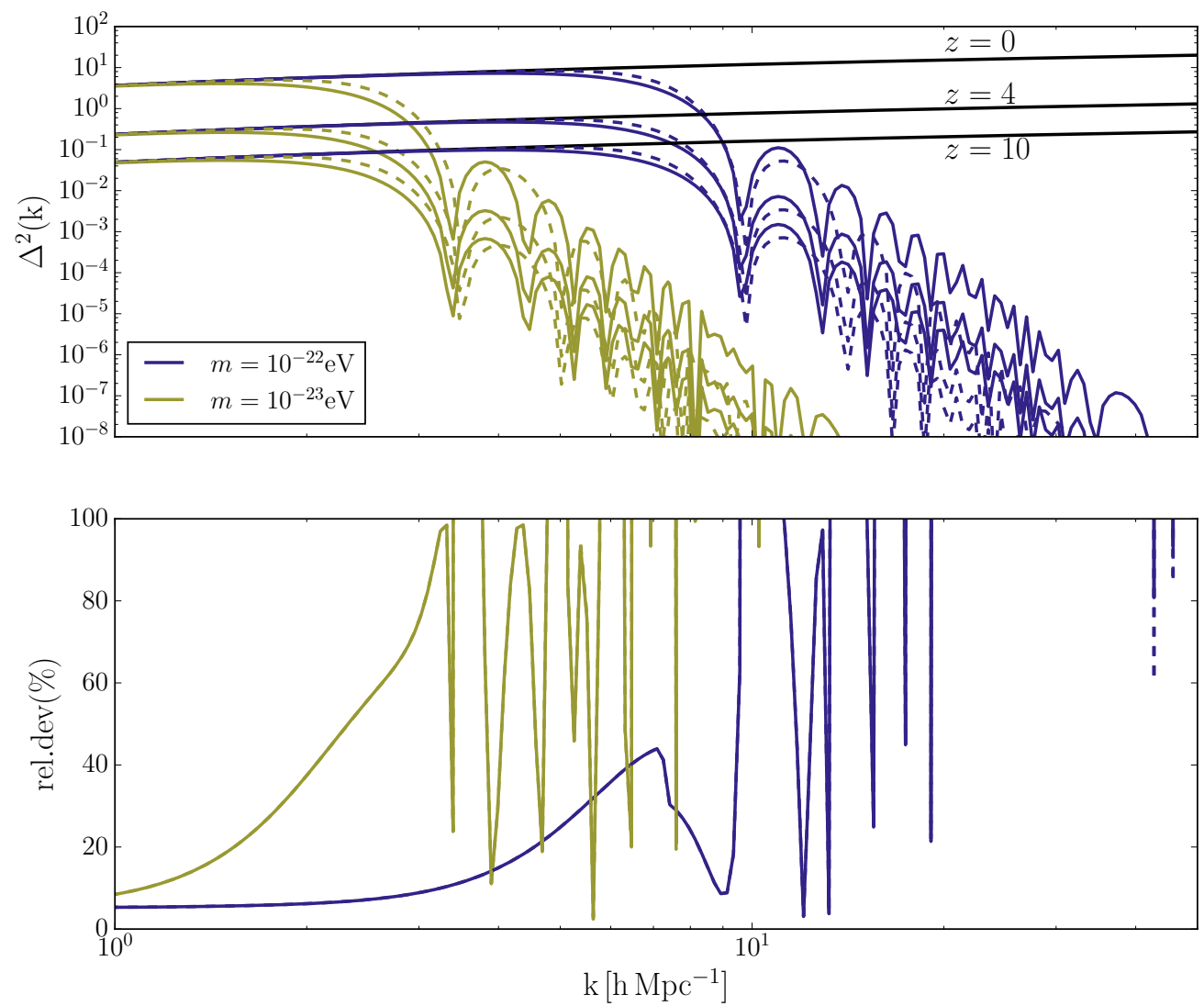

Figure 8. Dimensionless PS at low and medium redshifts for two different values of the scalar field mass, and their comparison with the approximation in Eq. 3.13. In the bottom panel we show the error between the two approaches, which is noticeably larger for smaller values of the scalar field mass. We only show here the oscillatory range in the PS, see Fig. 9 for the differences at small values of $k$.

smaller scales are dominated by the non-linear evolution of perturbations[86].

By having the mass PS at semi-linear scales we can make rough predictions about different observables. One of the most interesting quantities to compute is the halo mass function, which can give us information about the fraction of the density that have collapsed into self-gravitating structures of a given mass. It is also the base function to have an estimation of the number of structures of a certain mass that can be used to set constraints on cosmological parameters through the evolution of the number of clusters[87], or to infer properties about the dark matter through the amount of expected Milky Way satellites [88]. Even though for these observations the semi-analytic mass function will only be an approximation since it includes non-linear physics only approximately . For observations at large redshift it could give more reliable information [89].

Previous studies of structure formation with small-scale suppression, particularly for WDM, have shown that the Top-hat model fails in capturing the behavior of the cut-off in 

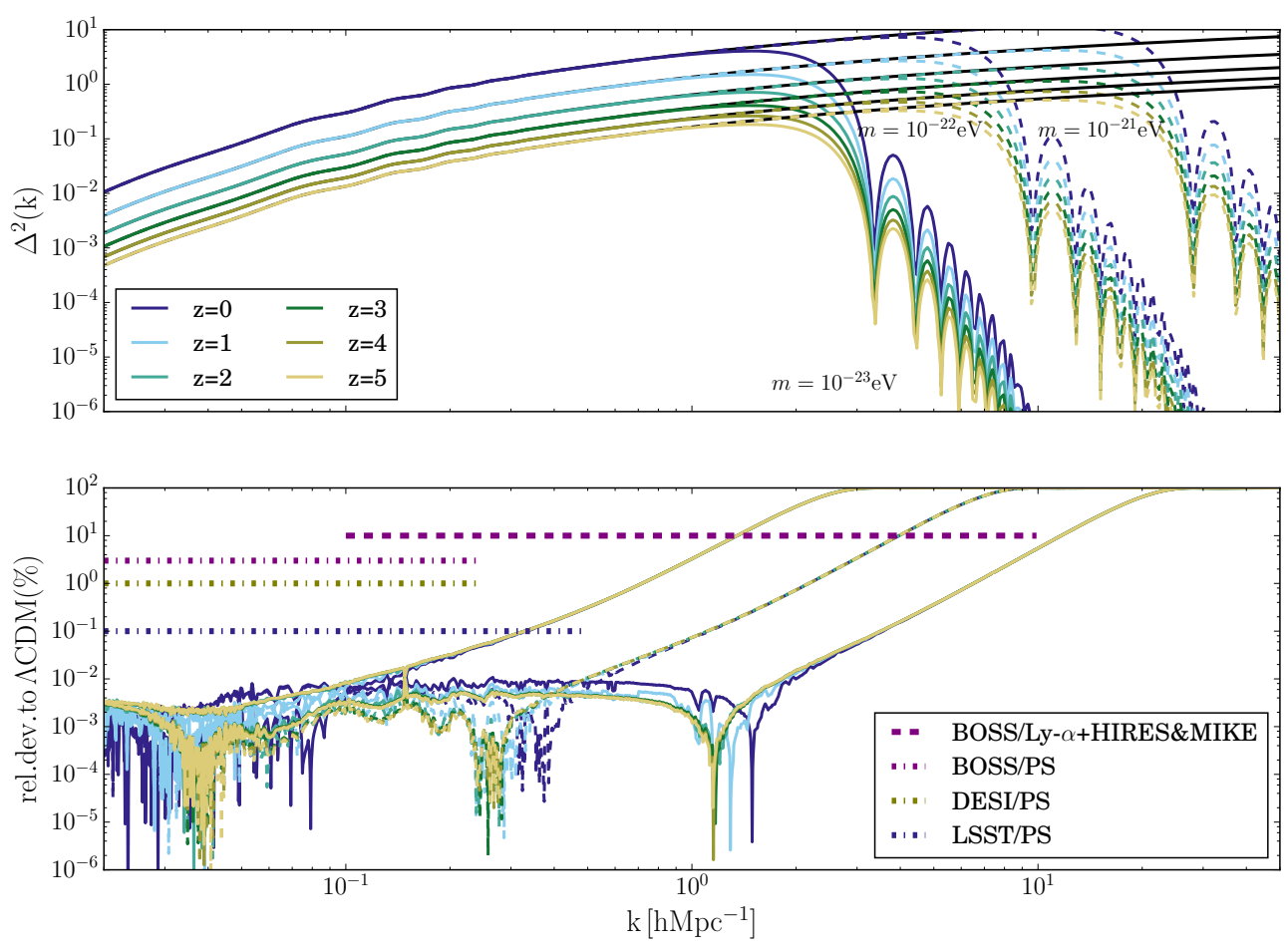

Figure 9. The same as in Fig. 8 but for the $k$-range that is usually tested with data from large scale galaxy and quasar surveys. A simple comparison shows that future LSST PS observations alone could place strong constraints in the mass range $m \gtrsim 10^{-23} \mathrm{eV}$. DESI and LSST in combination with other observables could constrain the $10^{-23}-10^{-22} \mathrm{eV}$ range .

the power spectrum, and how it translates into an almost complete suppression of the small scale structure. In contrast, the sharp- $k$ window function seems to better reproduce the mass function from simulations. Some examples of these effects were shown in[90-92]. The explanation provided in[91] is that with a sharp- $k$ filter the halo mass function depends on the shape of the PS for any radius, while for a Top-hat filter it becomes completely insensitive to the PS shape as soon as the latter decreases faster than $k^{3}$. More recently, the simulations for the SFDM model done by [84] also showed the presence of a turn around in the halo mass function that seems to be better described by a sharp- $k$ function than by the standard Tophat one. In Fig. 10, we show the halo mass function of SFDM computed as in[88, 91], where a semi-analytic approach based on the original Press-Schechter methodology is presented in the context of WDM. We used a Top-hat window function, and a sharp- $k$ function, for a scalar field mass of $m=10^{-22} \mathrm{eV}$. We also computed the mass function using a mass dependent barrier following [39] (see also a recent implementation in[93]). We show for comparison the halo mass function fit found from the numerical simulations in[84]. We can note that the small mass scales are very sensitive to the choice of the window function. Qualitatively, the sharp cut-off in the PS translates into a small suppression in the mass function if a Top-hat function is used, while it translates into a more accute cut-off for the sharp- $k$ function and the scale dependent growth factor. Since for the mass of our choice the cut-off happens at 


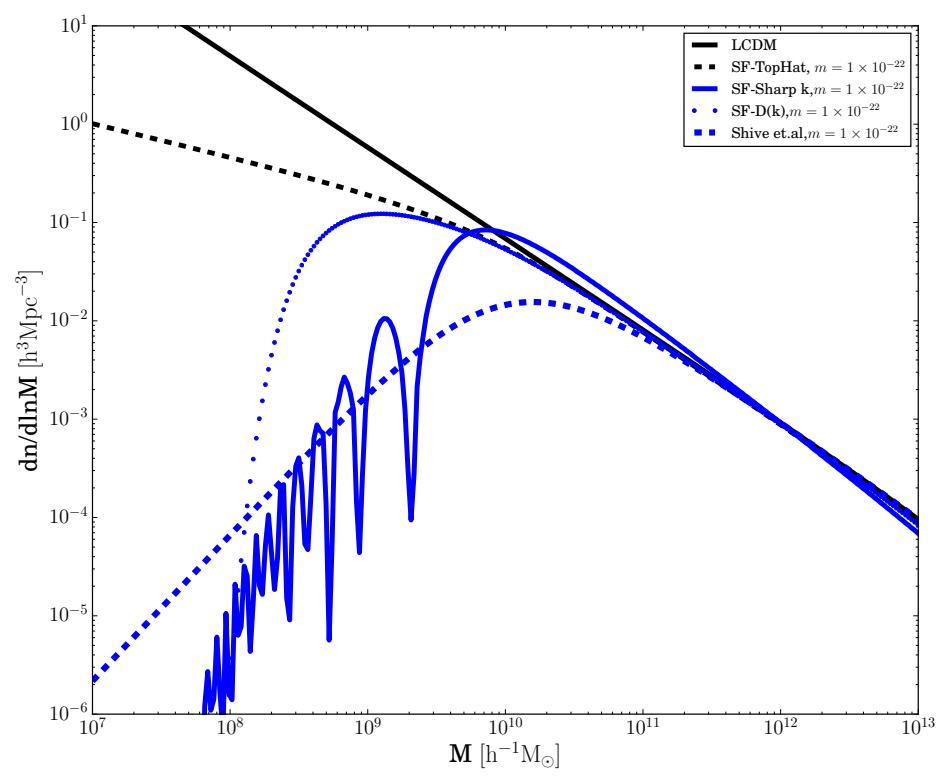

Figure 10. Logarithmic derivative of the halo mass function for $\Lambda \mathrm{CDM}$ and $\Lambda S F D M$ (with $m=10^{-22} \mathrm{eV}$ ). The cases depicted are the Top-hat (dashed curve), the Top-hat with a scale dependent growth function (dotted curve), the sharp- $k$ window function (solid curve), and the fit function inferred from the numerical simulations in[84]. The suppression of structures below $10^{10} \mathrm{~h}^{-1} \mathrm{M}_{\odot}$ is clear for all functions, although there are clear qualitative and quantitative differences among them. See the text for more details.

mass scales smaller than $10^{10}$ Solar masses, we still expect it to be consistent with current numbers of observed satellite galaxies in the local group. The cut-off mass we find with the sharp- $k$ window function captures well the result from the numerical simulations in terms of the scale at which the turn is happening, although there is a significant difference in the amplitude for the parameters we used. Note that we set the the sharp- $k$ function to match $\Lambda \mathrm{CDM}$ at large masses.

Finally, it is worth to mention that both window functions, Top-hat or sharp- $k$, need the input from numerical simulations of structure formation for a reliable computation of the halo mass function. The sharp- $k$ function needs it to set the mass scale at the turn-around, while the Top-hat needs it to set the $q$-value, which formally is a free parameter, in the first-crossing distribution. In[91], the mass at the PS turn around was fixed using warm dark matter simulations, and it was argued that this should be independent of the dark matter model, as soon as the cut-off is present. We consider it should be fixed by simulations of scalar field dark matter in order to not introduce a potential source of error. From the comparison in Fig. 10 among the different window functions and the functional form proposed in[84], it seems that the sharp- $k$ window function, is the one that is capturing better the non-linear effects, found in the simulations. However in such figure we just set the free parameters in order to have a reasonable match of the halo mass function to the $\Lambda \mathrm{CDM}$ one at large mass scales. Some work about how the differences in the halo mass function propagate to the number of satellites in a Milky Way type galaxy can be found in[19]. 


\section{Conclusions}

We have presented a new method to transform and solve numerically the equations of motion of a scalar field as a dark matter component. This method takes advantage of the explicit symmetries of the coupled Einstein-Klein-Gordon system of equations, and allow a clear understanding of the underlying physical solutions of the scalar field. The method is also suitable for numerical purposes, and deals correctly with the rapid oscillations of the field without any pre-handling of the equations of motion.

We showed that the background dynamics of SFDM is very simple in terms of the new dynamical variables, and even eases its numerical implementation in CMB codes, as exemplified with the results obtained from CLASS. Moreover, the transformation of variables used for the background case was easily adapted for the evolution of linear perturbations in the scalar field, and there was no need to rely on any similarities with standard fluid dynamics. Under this approach, we were able to find semi-analytical solutions which show in a clear way the physical effects acting in the growing or suppression of linear perturbations. The amended version of CLASS will be used in future studies to determine the direct effects of scalar fields in different observables, specially on those that make the CMB spectrum distinguishable from that of $\Lambda \mathrm{CDM}$ (see Fig. 2), for both types of dark matter and dark energy fields. Another topic of interest is the setting up of initial conditions of $N$-body and wave-like simulations, so that one can incorporate constraints for scalar field models from linear and non-linear perturbations.

Our results indicate that the growing of perturbations in SFDM models for a given wave number $k$ requires of two conditions: a) an early onset of field oscillations, preferably much earlier than the time of radiation-matter equality, and b) a negligible ratio $k / k_{J}$. Not surprisingly, both conditions depend upon the only free physical parameter which is the mass of the SFDM particle, and actually the two conditions are simultaneously enhanced (suppressed) in the case of large (small) values of $m$ just because both angular variables $\theta$ and $\vartheta$ evolve similarly throughout.

As for condition b), we must recall first that the scales that have been affected at some degree by the Jeans wavenumber $k_{J}$, throughout the evolution of the Universe, are those for which initially $k / k_{J, e q}>1$ with $k_{J, e q}=\Omega_{r 0}^{1 / 4} k_{J 0}$, see Eq. (3.6b). From this, we can infer that suppression of power, as compared to the standard CDM case, happens in scales:

$$
\frac{k}{\mathrm{Mpc}}>0.68 \Omega_{r 0}^{-1 / 4}\left(\frac{m}{10^{-23} \mathrm{eV}}\right)^{1 / 2}\left(\frac{H}{\mathrm{~km} \mathrm{~s}^{-1} \mathrm{Mpc}^{-1}}\right)^{1 / 2} .
$$

This suggests, for instance, that for $m \geq 10^{-24} \mathrm{eV}$ even the slightest suppression of perturbations will lie at scales $k>1 \mathrm{Mpc}^{-1}$, an estimation that is in agreement with the numerical results shown in Fig. 2. In summary, for $m \geq 10^{-24} \mathrm{eV}$ the main source of suppression of SFDM linear perturbations is the Jeans length $k_{J}$, whereas for smaller values of $m$ linear perturbations are also suppressed by the late appearance of the scalar field oscillations in the background.

We have recovered the results for SFDM perturbations that have been already reported in the literature under the fluid-approximation of the equations of motion for perturbations[18, $22,39]$. However, we would like to emphasize that our method does not have a direct translation to the fluid-approximation, because some fluid quantities cannot be well defined in terms of our variables. An example here is the sound speed of linear perturbations, which in 
our variables would be written as:

$$
c_{s}^{2}=\frac{\delta p_{\phi}}{\delta \rho_{\phi}}=\frac{\sin (\theta+\tilde{\vartheta} / 2)}{\sin (\tilde{\vartheta} / 2)}=\sin \theta \tan (\tilde{\vartheta} / 2)+\cos \theta \simeq \sin (2 m t) \tan (\tilde{\vartheta} / 2)+\cos (2 m t),
$$

where the last expression on the rhs is the approximate one after the onset of the field oscillations, see also Eqs. (3.4). From our perspective, $c_{s}^{2}$ becomes a rapidly-oscillating function, and then our cut-off procedure would dictate that $c_{s}^{2} \rightarrow 0$ for both large and small scales. This is at variance with the fluid approximation used in previous papers[18, 22, 39], and seems also to be an early indication that a direct translation to the fluid notation may not be straightforward. For this reason, the connection of our variables to those of the fluid approximation is a topic that deserves further investigation, but we leave this issue for a future work.

Finally, we have explored some differences that appear in the mass PS with respect to previous results published in the literature. The wavenumber at with which the cut-off of the power spectrum appears is consistent with previously reported results [19, 84], but the suppression of power is not as sharp; this small differences can be significant for the determination of the mass function of small scale structure. The mass of the scalar field needs to be larger that $10^{-23} \mathrm{eV}$ in order to be compatible with current power spectrum measurements. Future LSST survey will probably be able to prove the region $m \gtrsim 10^{-23} \mathrm{eV}$ (competitive to e.g.[89] from reionization or to [94] from pulsar timing). A further analysis of these constraints will be presented elsewhere.

\section{Acknowledgments}

We want to thank Francisco Linares for a careful reading of the manuscript. We are grateful to an anonymous referee for enlightening comments and suggestions. LAU-L wishes to thank Andrew Liddle and the Royal Observatory, Edinburgh, for their kind hospitality in a fruitful sabbatical stay, where part of this work was done. AXGM acknowledges support from Cátedras CONACYT and UCMEXUS-CONACYT collaborative proyect funding. This work was partially supported by PRODEP, DAIP-UGTO research grant $732 / 2016$ and 878/2016, PIFI, CONACyT México under grants 232893 (sabbatical), 167335, 179881 and Fronteras 281, Fundación Marcos Moshinsky, and the Instituto Avanzado de Cosmología (IAC) collaboration.

\section{A Cut-off procedure for rapidly oscillating terms}

Even though the numerical solutions seem to be well behaved after the cut-off implemented on the rapidly oscillating functions that appear in the equations of motion, the question remains of whether the cut-off is erasing any important part of the expected solution. As we will show now, the full solutions of different SF quantities can be decomposed in a monotone solution plus rapidly oscillating terms that quickly disappear at late times after the onset of rapid oscillations.

\section{A.1 Background variables}

The simplest case is that of variable $\theta$ itself, for which there is an exact solution. From Sec. 2.2, we learned that Eq. (2.7a) can be written in terms of the cosmic time as:

$$
\dot{\theta}=2 m-3 H \sin \theta \text {. }
$$


It was shown that the second term on the rhs of Eq. (A.1) becomes negligible after the onset of rapid oscillations, and then the dominant solution at late times is $\theta(t)=2 m t$. The latter is also the solution that is obtained once we apply the cut-off on the rapidly oscillating term on the rhs of Eq. (A.1) at $\theta\left(t_{\star}\right)=\theta_{\star}$.

This suggests that an approximate solution after the cut-off can be obtained by the substitution of $2 m t$ on the rhs of Eq. (A.1), and then:

$$
\theta(t)=2 m t-3 \int_{t_{\star}}^{t} H \sin (2 m t) d t .
$$

The Hubble parameter is given by $H=p / t$, where $p=1 / 2(p=2 / 3)$ for radiation (matter) domination, so that Eq. (A.2a) can be rewritten as:

$$
\theta(t)=2 m t-3 p \int_{\theta_{\star}}^{\theta} \frac{\sin x}{x} d x=2 m t-3 p\left[\operatorname{Si}(\theta)-\operatorname{Si}\left(\theta_{\star}\right)\right],
$$

where $\operatorname{Si}(x)$ is the sine integral. Thus, the solution of $\theta(t)$ consists of the linearly-growing term $2 m t$, plus a rapidly oscillating function with a decaying amplitude. From the properties of the sine integral, we find that $\left[\operatorname{Si}(\theta)-\operatorname{Si}\left(\theta_{\star}\right)\right] / \theta<8.6 \times 10^{-5}$ if $\theta>\hat{\theta}_{\star}=10^{2}$. Thus, we can safely say that the cut-off procedure applied to Eq. (2.7a) is just eliminating a negligible part of the full solution of $\theta(t)$ at late times.

We now use the above result to obtain an approximate solution of the density parameter after the the rapidly oscillating terms of are cut-off. Eq. (2.7c) can be written as:

$$
d\left(\ln \Omega_{\phi}\right)=3 w_{t o t} d N+3 H \cos \theta d t \simeq 3 w_{t o t} d N+3 p \frac{\cos \theta}{\theta} d \theta
$$

where we have assumed that $\theta=2 m t$ for $t>t_{\star}$. Eq. (A.3) indicates the existence of two different time scales in the evolution of $\Omega_{\phi}$ : the small one corresponding to rapid oscillations and represented here by $\theta$, and the large one represented by the number of $e$-folds $N$. Upon integration, we find that:

$$
\begin{aligned}
\Omega_{\phi}(t) & =\Omega_{\phi \star} \exp \left[3 \int_{N_{\star}}^{N} w_{t o t} d x\right] \exp \left[3 p \int_{\theta_{\star}}^{\theta} \frac{\cos x}{x} d x\right], \\
& =\Omega_{\phi \star} \exp \left[3 \int_{N_{\star}}^{N} w_{t o t} d x\right] \exp \left[3 p\left(\operatorname{Ci}\left(\theta_{\star}\right)-\operatorname{Ci}(\theta)\right)\right],
\end{aligned}
$$

with $\mathrm{Ci}(\mathrm{x})$ being the cosine integral, and $\Omega_{*}=\Omega\left(t_{*}\right)$. As expected, $\Omega_{\phi}$ has a steady evolution provided by the integral in the first exponential term on the rsh of Eq. (A.4), with some superimposed oscillations that decay away very quickly, which are obtained from the cosine integral in the last exponential term. Such oscillations in $\Omega_{\phi}(t)$ can even be seen by eye, for example, in some of the numerical solutions shown in Fig. 1.

The amended version of CLASS gives the same result for the density parameter as that obtained directly from Eq. (2.7c) before the cut-off is applied to the rapidly oscillating functions. After this time, that is for $\theta>\theta_{\star}$, the solution provided by CLASS simply is:

$$
\Omega_{\phi}^{\mathrm{CLASS}}(t)=\Omega_{\phi \star} \exp \left[3 \int_{N_{\star}}^{N} w_{t o t} d x\right] .
$$


We can clearly see that the cut-off procedure is equivalent to putting away the superimposed oscillations in the solution of $\Omega_{\phi}$. A quick comparison between Eqs. (A.4) and (A.5) shows that for $\theta>\theta_{*}$

$$
\frac{\Omega_{\phi}(t)-\Omega_{\phi}^{\mathrm{CLASS}}(t)}{\Omega_{\phi}(t)}<3 p \operatorname{Ci}\left(\theta_{\star}\right)
$$

and then the relative error between the true and the amended numerical solutions depends solely on $\theta_{\star}$. For our empirical choice of $\theta_{\star}=10^{2}$, we find that $\operatorname{Ci}\left(10^{2}\right) \simeq-5.1 \times 10^{-3}$, which means that the relative error in the density parameter calculated from CLASS with respect to the true solution grows with time but is always less than $1 \%$.

\section{A.2 Numerical examples}
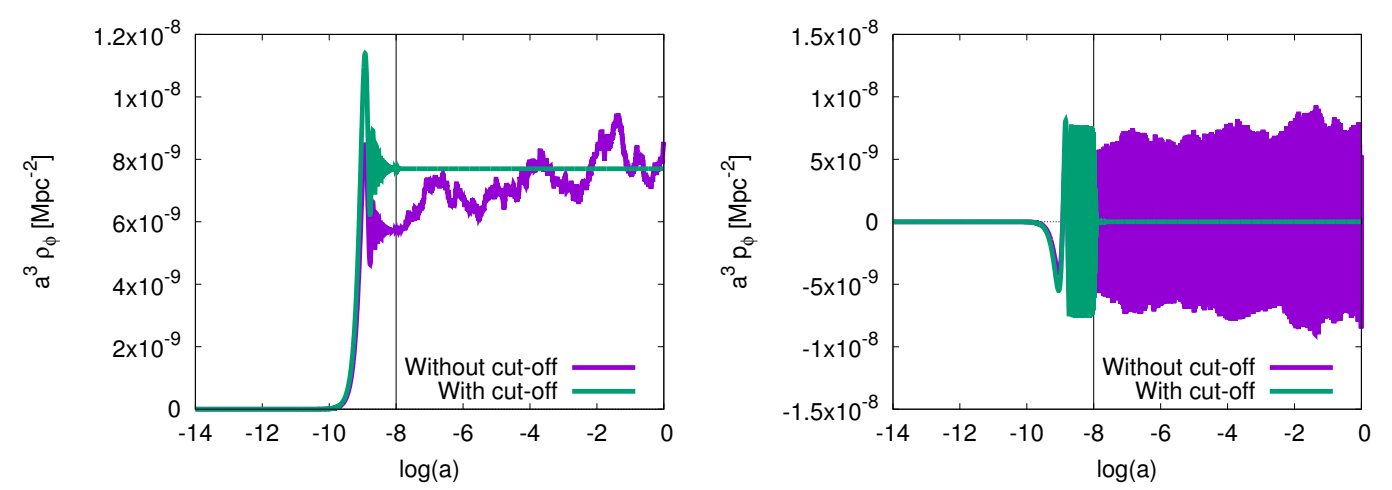

Figure 11. (Left) The normalized energy density $a^{3} \kappa^{2} \rho_{\phi} / 3$ in CLASS units, for a comparison between the cut and uncut cases of high frequency oscillations. We notice that the CLASS equation solver cannot give accurate numerical results for $a>10^{-8}$, but the cut-off case gives a smooth transition to the correct solution at late times. In this case, the empirical choice $\theta_{\star}=10^{2}$ means that the cut-off, indicated by the vertical line, is applied at $a=10^{-8}$. (Right) The normalized pressure $a^{3} \kappa^{2} p_{\phi} / 3$ for the cut and uncut cases. The cut-off in the oscillations is more drastic in this case, although it can be seen that the pressure oscillates a bit erratically around zero in the full numerical solution, which signals the limitations of CLASS when dealing with stiff systems of equations. See the text and Sec. 2.4 for more details.

In order to show the behavior of the numerical solutions under the cut-off procedure implemented for the trigonometric functions through Eq. (2.18), we present in Fig. 11 the cases of the scalar field energy density $\rho_{\phi}$ and pressure $p_{\phi}$; the examples correspond to the same Universes shown in Fig. 1 with a mass of $m=10^{-22} \mathrm{eV}$. First of all, it can be seen that the uncut case cannot be handled appropriately by the equation solver within CLASS, and the evolution of the scalar field variables appears as erratic after the onset of the rapid oscillations. One undesirable consequence of this is that the present contribution of the scalar field, as given by $\Omega_{\phi 0}$, cannot be determined accurately enough from the given initial conditions discussed in Sec. 2.3.

The evolution with the cut-off implemented, on the other hand, is far more stable, and, in the case of the energy density $\rho_{\phi}$, it matches smoothly the expected behavior at late times described in Secs. 2.2 and A.1. In contrast to the uncut case, the present scalar field contribution $\Omega_{\phi 0}$ is given exactly as desired, and also determined uniquely in terms of the initial conditions (see Eq. (2.15)). As for the scalar field pressure $p_{\phi}$, see Eq. (2.17), it is drastically affected by the sudden cut-off of the oscillations in the scalar field EoS $w_{\phi}$, but 
this does not have any major consequences for the overall evolution of all other cosmological variables. This again confirms that the cut-off procedure is a least invasive modification of the numerical computations, and then a complementary one to the method of averaged quantities in the fluid approximation.

\section{A.3 Linear perturbations}

For the case of the perturbation variables, we can follow the same procedure as before but with some subtleties. To begin with, we first recall that after the onset of rapid oscillations $\theta=2 m t$, see Secs. 3.1 and A.1. Then, we write Eqs. (4.1) in terms of the cosmic time in the form:

$$
\dot{\mathbf{P}}=H \mathbf{P}^{\prime}=\left[H \mathcal{M}_{0}(t)+\mathcal{M}_{1}(t)\right] \mathbf{P}+\frac{1}{2} h^{\prime}\left[H \mathbf{N}_{0}+\mathbf{N}_{1}(t)\right],
$$

where $H$ is the Hubble parameter, a prime denotes derivative with respect to the number of $e$-folds $N$, and

$$
\begin{aligned}
\mathbf{P} & =\left[\begin{array}{c}
e^{\alpha} \cos (\tilde{\vartheta} / 2) \\
e^{\alpha} \sin (\tilde{\vartheta} / 2)
\end{array}\right], \quad \mathcal{M}_{0}(t)=\frac{\omega}{2}\left[\begin{array}{cc}
0 & 1 \\
-1 & 0
\end{array}\right], \\
\mathcal{M}_{1}(t) & =-\frac{H}{2}\left[\begin{array}{ll}
-\omega \sin (2 m t) & 6 \sin (2 m t)-\omega \cos (2 m t) \\
-\omega \cos (2 m t) & 6 \cos (2 m t)+\omega \sin (2 m t)
\end{array}\right], \\
\mathbf{N}_{0} & =\left[\begin{array}{l}
0 \\
1
\end{array}\right], \quad \mathbf{N}_{1}(t)=H\left[\begin{array}{c}
\sin (2 m t) \\
-\cos (2 m t)
\end{array}\right] .
\end{aligned}
$$

In order to understand the general behavior of the solutions, we will again assume that $H=p / t=2 m p /(2 m t)=2 m p / \theta$, and then we write Eq. (A.7b) in the following differential form:

$$
d\left[e^{\mathcal{U}_{0}(N)} e^{\mathcal{U}_{1}(\theta)} \mathbf{P}\right]=\frac{h^{\prime}}{2} e^{\mathcal{U}_{0}(N)} e^{\mathcal{U}_{1}(\theta)} \mathbf{N}_{0} d N+\frac{h^{\prime}}{2} e^{\mathcal{U}_{0}(N)} e^{\mathcal{U}_{1}(\theta)} \mathbf{N}_{1}(\theta) d \theta,
$$

where $d N=H d t$, and

$$
\mathcal{U}_{0}(t)=\int \mathcal{M}_{0}(N) d N, \quad \mathcal{U}_{1}(\theta)=\int \mathcal{M}_{1}(\theta) d \theta .
$$

As in the case of Eq. (A.3), Eqs. (A.8) also indicate the presence of the two different time scales in the evolution of linear perturbations. In contrast to Eq. (A.8a), the differential equation that is solved by the amended version of CLASS after the application of the cut-off on the rapidly oscillating terms is:

$$
d\left[e^{\mathcal{U}_{0}(N)} \mathbf{P}^{\text {CLASS }}\right]=\frac{h^{\prime}}{2} e^{\mathcal{U}_{0}(N)} \mathbf{N}_{0} d N,
$$

which then deals only with the time scale represented by $N$. Its solution is:

$$
\mathbf{P}^{\mathrm{CLASS}}(N)=e^{-\mathcal{U}_{0}(N)} \mathbf{P}_{\star}+\frac{1}{2} \int_{N_{\star}}^{N} h^{\prime} e^{\left[\mathcal{U}_{0}(\hat{N})-\mathcal{U}_{0}(N)\right]} \mathbf{N}_{0} d \hat{N},
$$

where $\mathbf{P}_{\star}=\mathbf{P}\left(t_{*}\right)$. Actually, it can be easily verified that Eq. (A.9a) is equivalent to Eq. (4.4a), whereas we recover Eq. (4.4b) from Eq. (A.9b) under the assumption that $\omega=$ const. 
Like in the case of the background variables, we only need to show that the integration of the rapidly oscillating terms in the general solution of (A.8a) have become irrelevant by the time the cut-off procedure is applied to the equations of motion. We first take a look at Eqs. (A.8b). The integration of the matrix function $\mathcal{M}_{1}$ involves the use of the following cosine and sine integrals:

$$
\begin{aligned}
\int_{t_{\star}}^{t} H \cos (2 m t) d t & =p \int_{\theta_{\star}}^{\theta} \frac{\cos x}{x} d x=p\left[\operatorname{Ci}\left(\theta_{\star}\right)-\operatorname{Ci}(\theta)\right], \\
\int_{t_{\star}}^{t} H \sin (2 m t) d t & =p \int_{\theta_{\star}}^{\theta} \frac{\sin x}{x} d x=p\left[\operatorname{Si}(\theta)-\operatorname{Si}\left(\theta_{\star}\right)\right], \\
\int_{t_{\star}}^{t} \omega H \cos (2 m t) d t & =\frac{k^{2}}{2 m} \int_{\theta_{\star}}^{\theta} \frac{\cos x}{a^{2}(x)} d x=\frac{\omega_{\star}}{2}\left[\operatorname{Ci}\left(\theta_{\star}\right)-\operatorname{Ci}(\theta)\right],
\end{aligned}
$$

where the last integral (A.10c) was made under the assumption of radiation domination only, and then $\omega_{\star}$ is the frequency $\omega$ at the time $t_{\star}$. As argued before for Eqs. (A.2b) and (A.5), because of our choice $\theta_{\star}=10^{2}$ and the presence of the cosine and sine integrals in Eqs. (A.10), we can see that the elements of the matrix $\mathcal{U}_{1}$ are already small enough (of the order of $10^{-2}$ ) when the cut-off procedure is applied.

This suggests that we can safely replace $e^{\mathcal{U}_{1}(\theta)} \simeq \mathcal{I}+\mathcal{O}\left(10^{-2}\right)$, where $\mathcal{I}$ is the unity matrix, and then Eq. (A.8a) can be written as:

$$
\mathbf{P}(N, \theta) \simeq \mathbf{P}^{\mathrm{CLASS}}(N)+\frac{1}{2} e^{-\mathcal{U}_{0}(N)} \int_{\theta_{\star}}^{\theta} h^{\prime} e^{\mathcal{U}_{0}(N)} \mathbf{N}_{1}(\theta) d \theta, \quad \mathbf{N}_{1}(\theta)=p\left[\begin{array}{c}
\sin (\theta) / \theta \\
-\cos (\theta) / \theta
\end{array}\right],
$$

where $\mathbf{P}^{\text {CLASS }}(N)$ is the solution in Eq. (A.9b). Even though the second term on the rhs of Eq. (A.11) still is a rapidly oscillating function, it cannot be discarded as simply as others due to the presence of $h^{\prime}$.

The last integral in Eq. (A.11) can be integrated iteratively by parts, and in such case it can be proved that if we retain the dominant term in the integrations we obtain ${ }^{12}$ :

$$
\mathbf{P}(N, \theta) \simeq \mathbf{P}^{\operatorname{CLASS}}(N)+\frac{h^{\prime}}{2} \mathbf{N}_{2}(\theta), \quad \mathbf{N}_{2}(\theta)=p\left[\begin{array}{c}
\operatorname{Si}(\theta)-\operatorname{Si}\left(\theta_{\star}\right) \\
\operatorname{Ci}(\theta)-\operatorname{Ci}\left(\theta_{\star}\right)
\end{array}\right] .
$$

We can apply here the same kind of trick we used in Sec. 4.2, where we inferred that for the growing case: $\mathbf{P}^{\text {CLASS }} \sim h^{\prime} / 2$, see Eq. (4.4b). Thus, the second term on the rhs of Eq. (A.12) adds a negligible oscillating function to the general solution; in other words, we have just found that $\mathbf{P}(N, \theta) \simeq \mathbf{P}^{C L A S S}(N)\left(1+\mathcal{O}\left(10^{-2}\right)\right)$, which is the same level of accuracy attained for the background variables.

\section{References}

[1] Planck Collaboration Collaboration, P. Ade et al., Planck 2015. XX. Constraints on inflation, arXiv: 1502.02114.

[2] M. Vogelsberger, S. Genel, V. Springel, P. Torrey, D. Sijacki, D. Xu, G. F. Snyder, D. Nelson, and L. Hernquist, Introducing the Illustris Project: Simulating the coevolution of dark and visible matter in the Universe, Mon. Not. Roy. Astron. Soc. 444 (2014), no. 2 1518-1547, [arXiv: 1405.2921].

\footnotetext{
${ }^{12}$ Interestingly enough, the solution (A.11) can be obtained by means of a direct integration of Eq. (A.8a) if $N$ and $\theta$ are considered independent variables. This would be a reasonable short-cut to get Eq. (A.8a) given that the variables indeed represent two completely different time scales in the general solution.
} 
[3] S. Genel, M. Vogelsberger, V. Springel, D. Sijacki, D. Nelson, G. Snyder, V. Rodriguez-Gomez, P. Torrey, and L. Hernquist, Introducing the Illustris Project: the evolution of galaxy populations across cosmic time, Mon. Not. Roy. Astron. Soc. 445 (2014), no. 1 175-200, [arXiv: 1405.3749].

[4] G. Bertone and D. Hooper, A History of Dark Matter, arXiv:1605.04909.

[5] Y. Yang, Constraints on the basic parameters of dark matter using the Planck data, Phys.Rev. D91 (2015), no. 8 083517, [arXiv: 1504.01195].

[6] C. Angrick, F. Pace, M. Bartelmann, and M. Roncarelli, Reaching agreement between cosmological parameters inferred from galaxy clusters and Planck, arXiv:1504.03187.

[7] J. Barranco, A. Bernal, and D. Nunez, Dark matter equation of state from rotational curves of galaxies, Mon.Not.Roy.Astron.Soc. 449 (2015), no. 1 403-413, [arXiv:1301.6785].

[8] A. Cuoco, J.-Q. Xia, M. Regis, E. Branchini, N. Fornengo, et al., Dark matter searches in the gamma-ray extragalactic background via cross-correlations with galaxy catalogues, arXiv: 1506.01030.

[9] PICO Collaboration, C. Amole et al., Dark Matter Search Results from the PICO-60 $\mathrm{CF}_{3} I$ Bubble Chamber, Submitted to: Phys. Rev. D (2015) [arXiv:1510.07754].

[10] V. Gluscevic, M. I. Gresham, S. D. McDermott, A. H. G. Peter, and K. M. Zurek, Identifying the Theory of Dark Matter with Direct Detection, arXiv:1506.04454.

[11] A. Ringwald, The hunt for axions, arXiv:1506.04259.

[12] S.-J. Sin, Late time cosmological phase transition and galactic halo as Bose liquid, Phys.Rev. D50 (1994) 3650-3654, [hep-ph/9205208].

[13] S. Ji and S. Sin, Late time phase transition and the galactic halo as a bose liquid: 2. The Effect of visible matter, Phys.Rev. D50 (1994) 3655-3659, [hep-ph/9409267].

[14] T. Matos and L. A. Urena-Lopez, Quintessence and scalar dark matter in the universe, Class.Quant.Grav. 17 (2000) L75-L81, [astro-ph/0004332].

[15] T. Matos and L. A. Urena-Lopez, A Further analysis of a cosmological model of quintessence and scalar dark matter, Phys.Rev. D63 (2001) 063506, [astro-ph/0006024].

[16] T. Matos, A. Vazquez-Gonzalez, and J. Magana, $\phi^{2}$ as Dark Matter, Mon.Not.Roy.Astron.Soc. 393 (2009) 1359-1369, [arXiv:0806.0683].

[17] V. Sahni and L.-M. Wang, A New cosmological model of quintessence and dark matter, Phys.Rev. D62 (2000) 103517, [astro-ph/9910097].

[18] D. J. Marsh and P. G. Ferreira, Ultra-Light Scalar Fields and the Growth of Structure in the Universe, Phys.Rev. D82 (2010) 103528, [arXiv: 1009.3501].

[19] D. J. E. Marsh and J. Silk, A Model For Halo Formation With Axion Mixed Dark Matter, Mon.Not.Roy.Astron.Soc. 437 (2013) 2652, [arXiv:1307.1705].

[20] T.-P. Woo and T. Chiueh, High-Resolution Simulation on Structure Formation with Extremely Light Bosonic Dark Matter, Astrophys.J. 697 (2009) 850-861, [arXiv:0806.0232].

[21] J.-c. Hwang and H. Noh, Axion as a Cold Dark Matter candidate, Phys.Lett. B680 (2009) 1-3, [arXiv:0902.4738].

[22] C.-G. Park, J.-c. Hwang, and H. Noh, Axion as a cold dark matter candidate: low-mass case, Phys.Rev. D86 (2012) 083535, [arXiv: 1207.3124].

[23] J. Beyer and C. Wetterich, Small scale structures in coupled scalar field dark matter, arXiv: 1407.0141. 
[24] B. Li, T. Rindler-Daller, and P. R. Shapiro, Cosmological Constraints on Bose-Einstein-Condensed Scalar Field Dark Matter, Phys.Rev. D89 (2014), no. 8083536 , [arXiv:1310.6061].

[25] L. A. Urena-Lopez, Nonrelativistic approach for cosmological scalar field dark matter, Phys.Rev. D90 (2014), no. 2 027306, [arXiv:1310.8601].

[26] L. A. Urena-Lopez, Bose-Einstein condensation of relativistic Scalar Field Dark Matter, JCAP 0901 (2009) 014, [arXiv:0806.3093].

[27] A. P. Lundgren, M. Bondarescu, R. Bondarescu, and J. Balakrishna, Luke-warm dark matter: Bose-condensation of ultra-light particles, Astrophys.J. 715 (2010) L35, [arXiv:1001.0051].

[28] A. Aguirre and A. Diez-Tejedor, Asymmetric condensed dark matter, arXiv:1502.07354.

[29] L. Amendola and R. Barbieri, Dark matter from an ultra-light pseudo-Goldsone-boson, Phys.Lett. B642 (2006) 192-196, [hep-ph/0509257].

[30] E. J. Copeland, M. Sami, and S. Tsujikawa, Dynamics of dark energy, Int.J.Mod.Phys. D15 (2006) 1753-1936, [hep-th/0603057].

[31] D. J. E. Marsh, Axion Cosmology, arXiv:1510.07633.

[32] J. Magana, T. Matos, A. Suarez, and F. Sanchez-Salcedo, Structure formation with scalar field dark matter: the field approach, JCAP 1210 (2012) 003, [arXiv: 1204.5255].

[33] T. Matos, J.-R. Luevano, I. Quiros, L. A. Urena-Lopez, and J. A. Vazquez, Dynamics of Scalar Field Dark Matter With a Cosh-like Potential, Phys.Rev. D80 (2009) 123521, [arXiv:0906.0396].

[34] J. Barranco and A. Bernal, Constraining scalar field properties with boson stars as black hole mimickers, AIP Conf. Proc. 1396 (2011) 171-175, [arXiv:1108.1208].

[35] P.-H. Chavanis, Collapse of a self-gravitating Bose-Einstein condensate with attractive self-interaction, arXiv: 1604.05904.

[36] M. S. Turner, Coherent Scalar Field Oscillations in an Expanding Universe, Phys.Rev. D28 (1983) 1243.

[37] B. Ratra, Expressions for linearized perturbations in a massive scalar field dominated cosmological model, Phys.Rev. D44 (1991) 352-364.

[38] A. de la Macorra and G. Piccinelli, General scalar fields as quintessence, Phys.Rev. D61 (2000) 123503, [hep-ph/9909459].

[39] R. Hlozek, D. Grin, D. J. E. Marsh, and P. G. Ferreira, A search for ultra-light axions using precision cosmological data, arXiv:1410.2896.

[40] J. A. R. Cembranos, A. L. Maroto, and S. J. N. Jareo, Cosmological perturbations in coherent oscillating scalar field models, JHEP 03 (2016) 013, [arXiv: 1509.08819].

[41] M. Alcubierre, A. de la Macorra, A. Diez-Tejedor, and J. M. Torres, Cosmological scalar field perturbations can grow, arXiv:1501.06918.

[42] L. Martinez-Medina, H. L. Bray, and T. Matos, On wave dark matter in spiral and barred galaxies, arXiv:1505.07154.

[43] L. Martinez-Medina, V. Robles, and T. Matos, Dwarf galaxies in multistate scalar field dark matter halos, Phys.Rev. D91 (2015), no. 2 023519, [arXiv:1410.4163].

[44] V. H. Robles, L. Martinez-Medina, and T. Matos, SFDM: A new formation mechanism of tidal debris, arXiv:1503.00799.

[45] V. H. Robles, V. Lora, T. Matos, and F. Sanchez-Salcedo, Evolution of a dwarf satellite galaxy embedded in a scalar field dark matter halo, arXiv:1404.3424. 
[46] V. Robles and T. Matos, Strong Lensing with Finite Temperature Scalar Field Dark Matter, Phys.Rev. D88 (2013) 083008, [arXiv: 1302.5944].

[47] A. X. Gonzalez-Morales, A. Diez-Tejedor, L. A. Urena-Lopez, and O. Valenzuela, Hints on halo evolution in scalar field dark matter models with galaxy observations, Phys.Rev. D87 (2013), no. 2 021301, [arXiv:1211.6431].

[48] A. Diez-Tejedor, A. X. Gonzalez-Morales, and S. Profumo, Dwarf spheroidal galaxies and Bose-Einstein condensate dark matter, Phys.Rev. D90 (2014), no. 4 043517, [arXiv: 1404.1054].

[49] T. Rindler-Daller and P. R. Shapiro, Angular Momentum and Vortex Formation in Bose-Einstein-Condensed Cold Dark Matter Haloes, Mon.Not.Roy.Astron.Soc. 422 (2012) 135-161, [arXiv:1106.1256].

[50] T. Rindler-Daller and P. R. Shapiro, Complex scalar field dark matter on galactic scales, Mod.Phys.Lett. A29 (2014), no. 2 1430002, [arXiv:1312.1734].

[51] H.-Y. Schive, T. Chiueh, and T. Broadhurst, Cosmic Structure as the Quantum Interference of a Coherent Dark Wave, arXiv:1406.6586.

[52] K. Enqvist, S. Nadathur, T. Sekiguchi, and T. Takahashi, Decaying dark matter and the tension in $\sigma_{8}$, arXiv: 1505.05511.

[53] C. Boehm, A. Riazuelo, S. H. Hansen, and R. Schaeffer, Interacting dark matter disguised as warm dark matter, Phys.Rev. D66 (2002) 083505, [astro-ph/0112522].

[54] A. Mead and J. Peacock, Remapping dark matter halo catalogues between cosmological simulations, Mon.Not.Roy.Astron.Soc. 440 (2014), no. 2 1233-1247, [arXiv:1308.5183].

[55] A. Mead, J. Peacock, L. Lombriser, and B. Li, Rapid simulation rescaling from standard to modified gravity models, arXiv:1412.5195.

[56] L. Lombriser, F. Simpson, and A. Mead, Unscreening modified gravity in the matter power spectrum, arXiv:1501.04961.

[57] L. A. Urena-Lopez, Unveiling the dynamics of the universe, physics/0609181.

[58] V. Faraoni and C. S. Protheroe, Scalar field cosmology in phase space, Gen.Rel.Grav. 45 (2013) 103-123, [arXiv:1209.3726].

[59] R. Garca-Salcedo, T. Gonzalez, F. A. Horta-Rangel, I. Quiros, and D. Sanchez-Guzmn, Introduction to the application of the dynamical systems theory in the study of the dynamics of cosmological models of dark energy, Eur.J.Phys. 36 (2015) 025008, [arXiv:1501.04851].

[60] J. Lesgourgues, The Cosmic Linear Anisotropy Solving System (CLASS) I: Overview, arXiv:1104.2932.

[61] E. J. Copeland, A. R. Liddle, and D. Wands, Exponential potentials and cosmological scaling solutions, Phys.Rev. D57 (1998) 4686-4690, [gr-qc/9711068].

[62] M. J. Reyes-Ibarra and L. A. Urena-Lopez, Attractor dynamics of inflationary monomial potentials, AIP Conf.Proc. 1256 (2010) 293-310.

[63] L. A. Urena-Lopez, New perturbative method for analytical solutions in single-field models of inflation, arXiv:1512.07142.

[64] A. D. Rendall, Late-time oscillatory behaviour for self-gravitating scalar fields, Class.Quant.Grav. 24 (2007) 667-678, [gr-qc/0611088].

[65] A. Alho and C. Uggla, Global dynamics and inflationary center manifold and slow-roll approximants, arXiv:1406.0438.

[66] J. F. Jesus, J. L. G. Malatrasi, and S. H. Pereira, Can Dark Matter be a Scalar Field?, arXiv: 1504.04037. 
[67] D. Blas, J. Lesgourgues, and T. Tram, The Cosmic Linear Anisotropy Solving System (CLASS) II: Approximation schemes, JCAP 1107 (2011) 034, [arXiv:1104.2933].

[68] J. Lesgourgues, The Cosmic Linear Anisotropy Solving System (CLASS) III: Comparision with CAMB for LambdaCDM, arXiv:1104.2934.

[69] J. Martin, The Observational Status of Cosmic Inflation after Planck, arXiv:1502.05733.

[70] P. G. Ferreira and M. Joyce, Structure formation with a selftuning scalar field, Phys.Rev.Lett. 79 (1997) 4740-4743, [astro-ph/9707286].

[71] P. G. Ferreira and M. Joyce, Cosmology with a primordial scaling field, Phys.Rev. D58 (1998) 023503, [astro-ph/9711102].

[72] F. Perrotta and C. Baccigalupi, Early time perturbations behavior in scalar field cosmologies, Phys.Rev. D59 (1999) 123508, [astro-ph/9811156].

[73] H. Noh, C.-G. Park, and J.-c. Hwang, Axion as a Cold Dark Matter Candidate: Proof to Second order, Phys.Lett. B726 (2013) 559-563, [arXiv:1309.5692].

[74] D. J. E. Marsh, Non-linear hydrodynamics of axion dark matter: relative velocity effects and "quantum forces", arXiv:1504.00308.

[75] W. Hu, Structure formation with generalized dark matter, Astrophys.J. 506 (1998) 485-494, [astro-ph/9801234].

[76] W. Hu, R. Barkana, and A. Gruzinov, Cold and fuzzy dark matter, Phys.Rev.Lett. 85 (2000) 1158-1161, [astro-ph/0003365].

[77] C.-P. Ma and E. Bertschinger, Cosmological perturbation theory in the synchronous and conformal Newtonian gauges, Astrophys. J. 455 (1995) 7-25, [astro-ph/9506072].

[78] A. J. Christopherson, J. C. Hidalgo, and K. A. Malik, Modelling non-dust fluids in cosmology, JCAP 1301 (2013) 002, [arXiv:1207.1870].

[79] Planck Collaboration, P. Ade et al., Planck 2015 results. XIII. Cosmological parameters, arXiv: 1502.01589.

[80] DESI Collaboration, M. Levi et al., The DESI Experiment, a whitepaper for Snowmass 2013, arXiv: 1308.0847.

[81] LSST Collaboration, Z. Ivezic, J. A. Tyson, R. Allsman, J. Andrew, and R. Angel, LSST: from Science Drivers to Reference Design and Anticipated Data Products, arXiv:0805.2366.

[82] A. Schneider, R. Teyssier, D. Potter, J. Stadel, J. Onions, D. S. Reed, R. E. Smith, V. Springel, and F. R. Pearce, Matter power spectrum and the challenge of percent accuracy, arXiv: 1503.05920.

[83] M. Viel, G. D. Becker, J. S. Bolton, and M. G. Haehnelt, Warm dark matter as a solution to the small scale crisis: New constraints from high redshift Lyman-forest data, Phys. Rev. D88 (2013) 043502, [arXiv: 1306.2314].

[84] H.-Y. Schive, T. Chiueh, T. Broadhurst, and K.-W. Huang, Contrasting Galaxy Formation from Quantum Wave Dark Matter, $\psi D M$, with $\Lambda C D M$, using Planck and Hubble Data, arXiv: 1508.04621.

[85] H. Zhan, L. Knox, A. Tyson, and V. Margoniner, Exploring large-scale structure with billions of galaxies, Astrophys. J. 640 (2006) 8-17, [astro-ph/0508119].

[86] LSST Science Collaboration, P. A. Abell, J. Allison, S. F. Anderson, J. R. Andrew, J. R. P. Angel, L. Armus, D. Arnett, S. J. Asztalos, T. S. Axelrod, and et al., LSST Science Book, Version 2.0, ArXiv e-prints (Dec., 2009) [arXiv: 0912.0201]. 
[87] D. S. Reed, R. E. Smith, D. Potter, A. Schneider, J. Stadel, and B. Moore, Towards an accurate mass function for precision cosmology, Mon. Not. Roy. Astron. Soc. 431 (May, 2013) 1866-1882, [arXiv:1206.5302].

[88] A. Schneider, R. E. Smith, and D. Reed, Halo Mass Function and the Free Streaming Scale, Mon. Not. Roy. Astron. Soc. 433 (2013) 1573, [arXiv:1303.0839].

[89] B. Bozek, D. J. E. Marsh, J. Silk, and R. F. G. Wyse, Galaxy UV-luminosity function and reionization constraints on axion dark matter, Mon. Not. Roy. Astron. Soc. 450 (2015), no. 1 209-222, [arXiv:1409.3544].

[90] A. J. Benson, A. Farahi, S. Cole, L. A. Moustakas, A. Jenkins, M. Lovell, R. Kennedy, J. Helly, and C. Frenk, Dark matter halo merger histories beyond cold dark matter - I. Methods and application to warm dark matter, Mon.Not.Roy.Astron.Soc. 428 (Jan., 2013) 1774-1789, [arXiv: 1209.3018].

[91] A. Schneider, Structure formation with suppressed small-scale perturbations, Mon. Not. Roy. Astron. Soc. 451 (2015), no. 3 3117-3130, [arXiv:1412.2133].

[92] M. R. Buckley, J. Zavala, F.-Y. Cyr-Racine, K. Sigurdson, and M. Vogelsberger, Scattering, Damping, and Acoustic Oscillations: Simulating the Structure of Dark Matter Halos with Relativistic Force Carriers, Phys. Rev. D90 (2014), no. 4 043524, [arXiv:1405.2075].

[93] D. J. E. Marsh, WarmAndFuzzy: the halo model beyond CDM, arXiv:1605.05973.

[94] A. Khmelnitsky and V. Rubakov, Pulsar timing signal from ultralight scalar dark matter, JCAP 1402 (2014) 019, [arXiv: 1309.5888]. 